\title{
A SUB-ARCSECOND SURVEY TOWARD CLASS 0 PROTOSTARS IN PERSEUS: SEARCHING FOR SIGNATURES OF PROTOSTELLAR DISKS
}

\author{
John J. Tobin ${ }^{1,2,11}$, Leslie W. Looney ${ }^{3}$, David J. Wilner ${ }^{4}$, Woojin Kwon ${ }^{5}$, Clatre J. Chandler ${ }^{6}$, Tyler L. Bourke ${ }^{4,7}$, \\ Laurent Loinard ${ }^{8}$, Hsin-Fang Chiang ${ }^{9}$, Scott Schnee $^{1}$, and XuePeng Chen ${ }^{10}$ \\ ${ }^{1}$ National Radio Astronomy Observatory, Charlottesville, VA 22903, USA \\ ${ }^{2}$ Leiden Observatory, Leiden University, P.O. Box 9513, 2300-RA Leiden, The Netherlands; tobin@strw.leidenuniv.nl \\ ${ }^{3}$ Department of Astronomy, University of Illinois, Urbana, IL 61801, USA \\ ${ }^{4}$ Harvard-Smithsonian Center for Astrophysics, Cambridge, MA 02138, USA \\ ${ }^{5}$ SRON Netherlands Institute for Space Research, Landleven 12, 9747 AD, Groningen, The Netherlands \\ ${ }^{6}$ National Radio Astronomy Observatory, Socorro, NM 87801, USA \\ 7 Square Kilometer Array, Jodrell Bank, UK \\ ${ }^{8}$ Centro de Radioastronomía y Astrofísica, UNAM, Apartado Postal 3-72 (Xangari), 58089 Morelia, Michoacán, México \\ ${ }^{9}$ Institute for Astronomy and NASA Astrobiology Institute, University of Hawaii at Manoa, Hilo, HI 96720, USA \\ ${ }^{10}$ Purple Mountain Observatory, Chinese Academy of Sciences, 2 West Beijing Road, Nanjing 210008, China \\ Received 2014 November 26; accepted 2015 March 16; published 2015 May 28
}

\begin{abstract}
We present a $1.3 \mathrm{~mm}$ dust continuum survey toward nine Class 0 protostars and two Class I protostars in the Perseus molecular cloud, using CARMA with a resolution of $\sim 0.3$ (70 AU). This sample approximately doubles the number of Class 0 protostars observed with spatial resolutions $<100 \mathrm{AU}$ at millimeter wavelengths, enabling the presence of large protostellar disks and proto-binary systems to be probed. We have detected flattened structures with radii > 100 AU around two sources (L1448 IRS2 and Per-emb-14), and these sources may be strong disk candidates. Marginally resolved structures within $30^{\circ}$ of perpendicular to the outflow are found toward three protostars (L1448 IRS3C, IRAS 03282+3035, L1448C) and are considered disk candidates. Two others (L1448 IRS3B, IRAS 03292+3039) have complex resolved structures, possibly indicative of massive, fragmenting inner envelopes or disks; L1448 IRS3B also has evidence for a companion separated by 0.9 ( 210 AU). The candidate first hydrostatic core L1451-MMS is marginally resolved on $1^{\prime \prime}$ scales and the Class 0 protostar IC 348-MMS and does not have strong indications of resolved structure at any scale. The strong disk candidate sources were followed up with $\mathrm{C}^{18} \mathrm{O}(J=2 \rightarrow 1)$ observations; we detect velocity gradients that are consistent with the expected rotation axis, but without enough sensitivity to determine if it is Keplerian. We compare the observed visibility amplitudes to radiative transfer models of protostellar envelopes and disks. The visibility amplitude ratios show that a compact component (possibly a disk) is necessary for five of nine Class 0 sources. An envelope-only scenario cannot be ruled out for the other four Class 0 sources. We conclude that there is evidence for the formation of large disks in the Class 0 phase, but Class 0 disks likely have a range of radii and masses that depend on the initial conditions of their parent cores.
\end{abstract}

Key words: protoplanetary disks - stars: formation - stars: protostars - techniques: interferometric

\section{INTRODUCTION}

Stars form due to the gravitational collapse of dense condensations within molecular clouds, and the star formation process is what ultimately leads to the formation of solar systems and potentially life itself, as has transpired in our solar system. Therefore, the formation of the proto-planetary disk is a key step in the process of forming a planetary system. Moreover, disks may also play a role in the formation of binary and multiple-star systems via gravitational instability (e.g., Kratter et al. 2010); about one-third of all star systems and $50 \%$ of Sun-like star systems are found in binary or multiple systems with a typical separation of $\sim 50 \mathrm{AU}$ (Raghavan et al. 2010).

The formation of disks is thought to begin early in the star formation process, during the Class 0 phase of protostellar evolution (Andre et al. 1993). This is the earliest recognizable phase of the star formation process, characterized by a protostar surrounded by a dense envelope of gas and dust. The early formation of disks is inferred from the ubiquity of outflows and jets during the protostellar phase (e.g., Frank et al. 2014, and

\footnotetext{
${ }^{11}$ Hubble Fellow.
}

references therein). Disks form during the collapse of the starforming cloud via conservation of angular momentum (e.g., Ulrich 1976; Cassen \& Moosman 1981; Terebey et al. 1984); the angular momentum may derive from bulk cloud rotation or the net angular momentum of residual turbulent motions.

Disk formation, however, may not be as simple if the protostellar cloud is magnetized (even weakly; Allen et al. 2003; Mellon \& Li 2008; Li et al. 2013). The magnetic field will be dragged inward with the collapsing material, increasing the local field strength. The magnetic field then slows the rotation of the inner envelope by transporting angular momentum to the larger-scale envelope (Allen et al. 2003; Mellon \& Li 2008). This "magnetic braking" can slow the rotation efficiently enough to suppress disk formation, and this result has become known as the "magnetic braking catastrophe." More recently, a number of ways around the catastrophe have been found: reduction of magnetic field strengths via ohmic dissipation (Dapp \& Basu 2010), misaligned rotation axes and magnetic field directions (Joos et al. 2012), turbulence (Seifried et al. 2013), reconnection diffusion (Lazarian et al. 2012), and/or reduced ionization (hence less field coupling; Padovani et al. 2013). 
While the theoretical difficulty of disk formation is resolved somehow by nature, Class 0 disks remain poorly characterized observationally. There have been efforts to detect and resolve Class 0 disks with millimeter interferometers for the past 20 years. Possible signatures of disks have been detected via dust continuum emission (e.g., Chandler et al. 1995; Brown et al. 2000; Looney et al. 2000; Harvey et al. 2003; Jørgensen et al. 2009); however, these pioneering efforts were not able to uniquely identify disks due to lack of sensitivity and/or spatial resolution. Further muddying the waters, Maury et al. (2010) did not resolve structure consistent with disks having $R>100 \mathrm{AU}$, using data from the Plateau de Bure Interferometer with $\sim 0.5$ resolution. However, the Maury et al. (2010) sample was composed of two Very Low Luminosity Objects (IRAM 04191 and L1521F; Bourke et al. 2006; Dunham et al. 2006) and three typical Class 0 sources (L1527 IRS, L1448C, NGC 1333 IRAS2A). Another high-resolution study (0.3) with the Combined Array for Research in Millimeter-wave Astronomy (CARMA) toward the Class 0 protostellar system L1157-mm also failed to resolve a disk (Chiang et al. 2012).

Following these studies, the Class 0 protostar L1527 IRS (also in the Maury et al. sample) was found to have evidence for an edge-on disk in $3.8 \mu \mathrm{m}$ scattered light (Tobin et al. 2010a). Subsequent follow-up observations of this source led to the disk being resolved in the dust continuum, as well as confirmation of rotational support (Tobin et al. 2012, 2013). The mass of the protostar was measured to be $\sim 0.2 M_{\odot}$, about $20 \%$ of the surrounding envelope mass. Observations of L1527 with the Atacama Large Millimeter/submillimeter Array (ALMA) now show that the mass of the protostar is likely 0.3 $M_{\odot}$, with a rotationally supported radius of $54 \mathrm{AU}$ (Ohashi et al. 2014). Sakai et al. (2014a, 2014b) also found evidence for a chemical change at the interface between the envelope and disk using new data from ALMA. Evidence of rotationally supported Class 0 disks in other systems have now been found with ALMA (Murillo et al. 2013; Codella et al. 2014; Lindberg et al. 2014). Thus, the combined results of previous studies point toward a diversity of disk properties in the Class 0 phase and imply that large disks may form in some systems before the end of the Class 0 phase.

Despite these previous efforts, the number of Class 0 protostars observed with spatial resolutions better than $100 \mathrm{AU}$ is only $\sim 10$ at millimeter and submillimeter wavelengths. To further the characterization of Class 0 disks, we have observed a sample of nine Class 0 protostars and two Class I protostars in the Perseus molecular cloud $(D \sim 230$ pc; Hirota et al. 2008, 2011) using the CARMA array at $1.3 \mathrm{~mm}$ with a resolution of $\sim 0$ !3 (70 AU). The Perseus molecular cloud was chosen for this study because it is the nearest star-forming region with a large number of Class 0 protostars; the more nearby Taurus and Ophiuchus clouds do not have sufficiently large numbers of Class 0 protostars from which to draw a meaningful sample.

In this paper, we present the results from our survey, examining the dust continuum structures and visibility amplitudes, and compare to radiative transfer models. We will also present molecular line follow-up obtained toward some sources using CARMA and the Submillimeter Array (SMA), as well as the $\mathrm{CO}(J=2 \rightarrow 1)$ outflow emission when detected. We present the sample, observations, and data reduction in Section 2, the continuum and molecular line results are described in Section 3, and a comparison to radiative transfer models is presented in Section 4. The results are discussed in Section 5, and we present our summary and conclusions in Section 6.

\section{OBSERVATIONS}

\subsection{Sample Selection}

We have drawn our sample of nine Class 0 sources (Table 1) from the known protostars in the Perseus molecular clouds based on the various infrared and millimeter studies that have been conducted (e.g., Hirano et al. 1999; Looney et al. 2000; Enoch et al. 2009; Chen et al. 2010; Pineda et al. 2011; Schnee et al. 2012; Sadavoy et al. 2014). In addition, two Class I protostars were located adjacent to Class 0 sources and within the CARMA field of view. Enoch et al. (2009) classify 27 sources as Class 0 protostars; however, additional sources are now known from more recent millimeter observations (references above), and one source in the Enoch et al. (2009) survey was incorrectly associated with IRAC emission (Peremb-37). Including these additional sources brings the total number of Class 0 protostars in Perseus to $\sim 38$.

We chose sources that were not known to be close binaries $(<1000 \mathrm{AU})$ and had clearly defined outflow axes from CO observations or Spitzer IRAC imaging (e.g., Jørgensen et al. 2006). We also focused on sources that had not been previously observed with sensitive, high-resolution observations, aiming to increase the number of sources with highresolution observations. Good knowledge of the outflow axis is important because this is a proxy for the current angular momentum vector of the system. If a sufficiently large disk is present, it is expected to be elongated perpendicular to the outflow direction.

The Class 0 sources range in luminosity from $0.7 L_{\odot}$ to 9.2 $L_{\odot}$ (Enoch et al. 2009; Sadavoy et al. 2014), and the candidate FHSC L1451-MMS has a luminosity less than $0.05 L_{\odot}$ (Pineda et al. 2011). This range of luminosity is representative of the distribution of protostellar luminosities in Perseus, with only a few systems having luminosities in excess of $10 L_{\odot}$. The sources also sample a range of environments: wide binary systems, isolated cores within the cloud, members of the NGC 1333 cluster, the outskirts of the older IC 348 cluster, and the moderately active star-forming cloud L1448. This sample enables us to begin more thoroughly exploring the presence of disks from their dust continuum emission.

\subsection{CARMA Observations and Data Reduction}

CARMA is a heterogeneous interferometer array located in the Inyo Mountains of California. It is composed of $6 \times 10.4 \mathrm{~m}$ antennas, $9 \times 6.1 \mathrm{~m}$ antennas, making up the main array, and $8 \times 3.5 \mathrm{~m}$ antennas that are operated as an independent array or as part of the CARMA23 array. The observations were conducted in $\mathrm{B}$ and $\mathrm{C}$ configurations of the main array between 2012 December and 2013 November; see Table 2. B configuration has baseline lengths up to $\sim 1 \mathrm{~km}$, and $\mathrm{C}$ configuration has baseline lengths up to $\sim 0.35 \mathrm{~km}$. The respective angular resolutions of the configurations are $\sim 0.3$ and $\sim 0.9$. Three science targets were observed in each track, including a test source $0326+277$ to verify the millimeter seeing in B configuration.

The observations were conducted in a standard loop, bracketing the three science pointings (and test source in B- 
Table 1

Source List

\begin{tabular}{|c|c|c|c|c|c|c|c|c|c|}
\hline Source & $\begin{array}{l}\text { R.A. } \\
\text { (J2000) }\end{array}$ & $\begin{array}{l}\text { Decl. } \\
(\mathrm{J} 2000)\end{array}$ & $\begin{array}{l}\text { Envelope Mass } \\
\qquad\left(M_{\odot}\right)\end{array}$ & $\begin{array}{l}L_{\mathrm{bol}} \\
\left(L_{\odot}\right)\end{array}$ & $\begin{array}{c}L_{\text {submm }} / L_{\text {bol }} \\
(\%)\end{array}$ & $\begin{array}{l}T_{\text {bol }} \\
(\mathrm{K})\end{array}$ & $\begin{array}{c}\text { Outflow PA } \\
\left({ }^{\circ}\right)\end{array}$ & References & Other Names \\
\hline L1451-MMS & $3: 25: 10.25$ & $+30: 23: 55.0$ & $0.36(0.04)$ & $<0.05$ & $\ldots$ & $<30$ & 10 & 2,8 & Per-bolo-2 \\
\hline L1448 IRS2 & $3: 25: 22.41$ & $+30: 45: 13.3$ & $1.41(0.14)$ & $3.6(0.5)$ & $2.7(0.4)$ & $43(2)$ & 318 & $2,3,4,5$ & Per-emb-22 \\
\hline L1448 IRS3B & $3: 25: 36.38$ & $+30: 45: 14.7$ & $2.7(0.27)$ & $8.3(0.8)$ & $3.4(0.4)$ & $57(3)$ & 285 & $2,5,6$ & L1448 NB, Per-emb-33 \\
\hline L1448 IRS3C & $3: 25: 35.67$ & $+30: 45: 34.1$ & $1.1(0.11)$ & $1.4(0.1)$ & $7.8(0.9)$ & $22(1)$ & 308 & $1,2,5$ & L1448 NW \\
\hline L1448 IRS3A & $3: 25: 36.50$ & $+30: 45: 21.8$ & $0.72(0.07)$ & $4.3(3.7)$ & $\ldots$ & $90(18)$ & $155 ?$ & $1,2,3,6$ & L1448 NA, Per-emb-33 \\
\hline L1448C & $3: 25: 38.87$ & $+30: 44: 05.3$ & $1.87(0.19)$ & $9.2(1.3)$ & $1.9(0.3)$ & $47(2)$ & 339 & $1,2,3,5,11$ & L1448-mm, Per-emb-26 \\
\hline L1448C-S & $3: 25: 39.14$ & $+30: 43: 57.7$ & $1.87(0.19)^{\mathrm{a}}$ & $0.68(0.85)$ & $\ldots$ & $163(51)$ & 40 & $2,3,10$ & Per-emb-42 \\
\hline Per-emb-24 & $3: 28: 45.31$ & $+31: 05: 41.6$ & $0.19(0.02)$ & $0.43(0.01)$ & $\ldots$ & $67(10)$ & 101 & 1,3 & IRAS 03256+3055 \\
\hline Per-emb-14 & $3: 29: 13.55$ & $+31: 13: 58.1$ & $0.5(0.05)$ & $0.7(0.08)$ & $6(2)$ & $31(2)$ & 95 & $1,3,5$ & NGC 1333 IRAS4C \\
\hline $\begin{array}{l}\text { IRAS } 03282 \\
\quad+3035\end{array}$ & $3: 31: 20.94$ & $+30: 45: 30.2$ & $0.96(0.4)$ & $1.3(0.1)$ & $5.1(0.7)$ & $32(2)$ & 125 & $3,5,9$ & Per-emb-5 \\
\hline $\begin{array}{l}\text { IRAS } 03292 \\
\quad+3039\end{array}$ & $3: 32: 17.92$ & $+30: 49: 48.1$ & $2.9(0.1)$ & $0.9(0.1)$ & $9(1)$ & $27(1)$ & 127 & $2,3,7$ & Per8, Per-emb-2 \\
\hline IC 348-MMS & $3: 43: 57.06$ & $+32: 03: 04.8$ & $1.78(0.04)$ & $1.5(0.1)$ & $6.4(0.9)$ & $30(2)$ & 330 & $3,5,10$ & Per-emb-11 \\
\hline
\end{tabular}

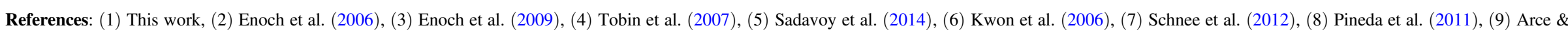

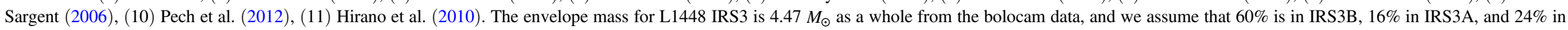
IRS3C. These ratios come from the ratio of flux at $30 \mathrm{k} \lambda$, for which the sources are all resolved from each other.

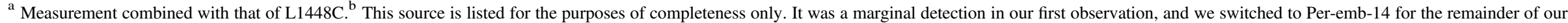
program. 
Table 2

CARMA 1.3 mm Observation Log

\begin{tabular}{|c|c|c|c|c|c|c|c|c|}
\hline Sources & Config. & $\begin{array}{l}\text { Date } \\
\text { (UT) }\end{array}$ & $\begin{array}{c}\text { Track } \\
\text { Length } \\
(\mathrm{hr})\end{array}$ & $\begin{array}{l}\text { Int. Time } \\
\text { (minutes) }\end{array}$ & $\begin{array}{l}\text { Central } \\
\text { Frequency } \\
\quad(\mathrm{GHz})\end{array}$ & $\begin{array}{l}\tau_{230 \mathrm{GHz}}, \mathrm{rms} \\
\text { Path, } T_{\text {sys }} \\
(\text { nepers, } \mu \mathrm{m}, \mathrm{K})\end{array}$ & $\begin{array}{c}\text { Gain Calibrator/Flux } \\
\text { Density } \\
\text { (Name, Jy) }\end{array}$ & Flux Calibrator \\
\hline L1448C & $\mathrm{C}$ & $\begin{array}{c}2012 \\
\text { Dec } 11\end{array}$ & 7.9 & 5.0 & 225.0491 & $0.21,153,350$ & $0237+288,1.6$ & Uranus \\
\hline $\begin{array}{l}\text { L1448 IRS3A, } \\
\text { IRS3B }\end{array}$ & $\cdots$ & $\cdots$ & $\cdots$ & $\cdots$ & $\cdots$ & $\cdots$ & $\cdots$ & $\cdots$ \\
\hline L1448 IRS3C & $\cdots$ & $\cdots$ & $\cdots$ & $\cdots$ & $\ldots$ & $\ldots$ & $\ldots$ & $\ldots$ \\
\hline L1448 IRS2 & $\mathrm{C}$ & $\begin{array}{c}2013 \\
\text { Jan } 04\end{array}$ & 4.5 & 5.0 & 225.0491 & $0.13,84,300$ & $0237+288,1.6$ & Uranus \\
\hline L1451-MMS & $\cdots$ & $\cdots$ & $\ldots$ & $\cdots$ & $\cdots$ & $\cdots$ & $\cdots$ & $\cdots$ \\
\hline Per-emb24 & $\cdots$ & $\cdots$ & $\cdots$ & $\cdots$ & $\ldots$ & $\ldots$ & $\ldots$ & $\cdots$ \\
\hline L1448 IRS2 & $\mathrm{C}$ & $\begin{array}{c}2013 \\
\text { Jan } 05\end{array}$ & 4.0 & 5.0 & 225.0491 & $0.23,124,400$ & $0237+288,1.6$ & Uranus \\
\hline L1451-MMS & $\ldots$ & $\ldots$ & $\ldots$ & $\cdots$ & $\cdots$ & $\cdots$ & $\ldots$ & $\ldots$ \\
\hline Per-emb14 & $\ldots$ & $\ldots$ & $\ldots$ & $\cdots$ & $\ldots$ & $\ldots$ & $\ldots$ & $\ldots$ \\
\hline L1448C & $\mathrm{B}$ & $\begin{array}{c}2013 \\
\text { Jan } 18\end{array}$ & 7.5 & 3.0 & 225.0491 & $0.08,95,250$ & $0237+288,1.3$ & Uranus \\
\hline $\begin{array}{l}\text { L1448 IRS3A, } \\
\text { IRS3B }\end{array}$ & $\cdots$ & $\ldots$ & $\cdots$ & $\cdots$ & $\cdots$ & $\cdots$ & $\ldots$ & \\
\hline L1448 IRS3C & $\ldots$ & $\ldots$ & $\ldots$ & $\ldots$ & $\ldots$ & $\cdots$ & $\ldots$ & \\
\hline $0326+277$ & $\cdots$ & $\ldots$ & $\cdots$ & 1.0 & $\ldots$ & $\ldots$ & $\ldots$ & $\ldots$ \\
\hline IRAS $03282+3035$ & $\mathrm{~B}$ & $\begin{array}{c}2013 \\
\text { Jan } 20\end{array}$ & 5.5 & 3.0 & 225.0491 & $0.075,66,200$ & $0237+288,1.3$ & Uranus \\
\hline IRAS 03292+3039 & $\cdots$ & $\cdots$ & $\cdots$ & $\ldots$ & $\cdots$ & $\cdots$ & $\cdots$ & $\cdots$ \\
\hline IC 348-MMS & $\cdots$ & $\cdots$ & $\cdots$ & $\cdots$ & $\cdots$ & $\cdots$ & $\cdots$ & $\cdots$ \\
\hline $0326+277$ & $\cdots$ & $\ldots$ & $\ldots$ & 1.0 & $\ldots$ & $\ldots$ & $\ldots$ & $\cdots$ \\
\hline IRAS $03282+3035$ & B & $\begin{array}{c}2013 \\
\text { Jan } 21\end{array}$ & 4.5 & 3.0 & 225.0491 & $0.08,82,250$ & $0237+288,1.3$ & Uranus \\
\hline IRAS 03292+3039 & $\cdots$ & $\cdots$ & $\cdots$ & $\cdots$ & $\cdots$ & $\cdots$ & $\cdots$ & $\cdots$ \\
\hline IC 348-MMS & $\cdots$ & $\cdots$ & $\cdots$ & $\cdots$ & $\cdots$ & $\cdots$ & $\cdots$ & $\cdots$ \\
\hline $0326+277$ & $\cdots$ & $\ldots$ & $\cdots$ & 1.0 & $\ldots$ & $\ldots$ & $\ldots$ & $\ldots$ \\
\hline L1448 IRS2 & B & $\begin{array}{c}2013 \\
\text { Jan } 24\end{array}$ & 7.0 & 3.0 & 225.0491 & $0.13,58,300$ & $0237+288,1.3$ & Uranus \\
\hline L1451-MMS & $\cdots$ & $\cdots$ & $\cdots$ & $\cdots$ & $\cdots$ & $\cdots$ & $\cdots$ & $\cdots$ \\
\hline Per-emb-14 & $\cdots$ & $\cdots$ & $\cdots$ & $\cdots$ & $\cdots$ & $\cdots$ & $\cdots$ & $\ldots$ \\
\hline $0326+277$ & $\cdots$ & $\cdots$ & $\cdots$ & 1.0 & $\ldots$ & $\ldots$ & $\ldots$ & $\cdots$ \\
\hline Per-emb-14 & $\mathrm{C}$ & $\begin{array}{c}2013 \\
\text { Nov } 16\end{array}$ & 4.0 & 10.0 & 225.0491 & $0.35,150,600$ & $0237+288,1.3$ & Uranus \\
\hline Per-emb-14 & $\cdots$ & $\begin{array}{c}2013 \\
\text { Nov } 17\end{array}$ & 4.0 & 10.0 & $\cdots$ & $0.27,142,400$ & $\cdots$ & $\ldots$ \\
\hline IRAS $03282+3035$ & $\mathrm{C}$ & $\begin{array}{c}2013 \\
\text { Nov } 18\end{array}$ & 4.5 & 5.0 & 225.0491 & $0.18,138,400$ & $0237+288,1.3$ & Uranus \\
\hline IRAS $03292+3039$ & $\ldots$ & $\cdots$ & $\ldots$ & $\ldots$ & $\ldots$ & $\cdots$ & $\cdots$ & $\cdots$ \\
\hline IC 348-MMS & $\cdots$ & $\cdots$ & $\cdots$ & $\cdots$ & $\cdots$ & $\cdots$ & $\cdots$ & $\cdots$ \\
\hline
\end{tabular}

configuration) with an observation of the phase calibrator $(0237+288)$. Flux (Uranus) and bandpass (3C 84) calibrators were observed at the beginning of each track. The C-PACS system had been used in previous extended configurations for correction of rapid phase variations (Pérez et al. 2010), but the system was not available in the 2012 and 2013 observing seasons. However, the data were taken during exceptionally good weather conditions, and standard calibration methods produced excellent data. The optical depth at $1.3 \mathrm{~mm}$ was typically $\sim 0.1$ during $\mathrm{B}$ configuration, and the rms path length (phase noise) was less than $100 \mu \mathrm{m}$ as measured by the phase monitor (see Table 2); $100 \mu \mathrm{m}$ rms path length corresponds to $\sim 0$.34 seeing, approximately the angular resolution provided by $\mathrm{B}$ configuration at $1.3 \mathrm{~mm}$.

The central frequency of our observations was $225.0491 \mathrm{GHz}(1.3 \mathrm{~mm})$, and the correlator was configured for single-polarization observations with six $500 \mathrm{MHz}$ subbands set for continuum observation, yielding $6 \mathrm{GHz}$ of continuum bandwidth (dual sideband). The remaining two spectral windows were configured for spectral line observation with $31 \mathrm{MHz}$ bandwidth and were set to observe the $(J=2 \rightarrow 1)$ transitions of ${ }^{12} \mathrm{CO},{ }^{13} \mathrm{CO}$, and $\mathrm{C}^{18} \mathrm{O}$; the individual channels were $97 \mathrm{kHz}$, corresponding to $\sim 0.13 \mathrm{~km}$ $\mathrm{s}^{-1}$ velocity resolution. Follow-up observations of Per-emb-14 in 2013 November used dual-polarization mode in order to have higher spectral line sensitivity.

We reduced and edited the visibility data using standard methods within the MIRIAD software package (Sault et al. 1995); see Tobin et al. (2013) or Chiang et al. (2012) for more details. The absolute flux calibration error is estimated to be $\sim 20 \%$. The data were imaged using the CLEAN algorithm using robust weighting to optimize the angular 
resolution and sensitivity. In order to ensure that our data reduction results in reliable images, we imaged the test source $0326+277$ for each B-configuration data set. Gaussian fits to the source demonstrate that it is consistent with a point source, verifying the good millimeter seeing.

\subsection{SMA Observations and Data Reduction}

The SMA is an eight-element interferometer array composed of $6.1 \mathrm{~m}$ antennas and is located on Mauna Kea in Hawaii. We observed the source L1448 IRS2 from the CARMA sample with the SMA in the Extended configuration on two dates in 2013 September (Table 3). We observed in two-receiver mode with the low-frequency receiver tuned to $225.434 \mathrm{GHz}(1.3 \mathrm{~mm})$ and the high-frequency receiver tuned to $351.135 \mathrm{GHz}(850 \mu \mathrm{m})$. In this mode, the correlator provides $4 \mathrm{GHz}$ of bandwidth $(2 \mathrm{GHz}$ in each of the upper and lower sidebands) from each receiver. Each $2 \mathrm{GHz}$ band is composed of 24 sub-bands with a bandwidth of $104 \mathrm{MHz}$, and our setup had 64 channels per sub-band by default, and we used high-resolution settings to observe $\mathrm{C}^{18} \mathrm{O}(J=2 \rightarrow 1)$ (1024 channels), ${ }^{13} \mathrm{CO}(J=2 \rightarrow 1)$ (512 channels), and ${ }^{12} \mathrm{CO}(J=2 \rightarrow 1)$ (512 channels) with the low-frequency receiver. With the high-frequency receiver, we observed the $\mathrm{H}^{13} \mathrm{CO}^{+}$and $\mathrm{HCO}^{+}(J=4 \rightarrow 3)$ transitions with 512 channels.

The visibility data were edited and calibrated using the MIR software package, an IDL-based software package originally developed for the Owens Valley Radio Observatory and adapted by the SMA group. The error in the absolute flux calibration is estimated to be $\sim 10 \%$. The calibrated visibilities were exported to MIRIAD format for imaging. We detected all the $\mathrm{CO}(J=2 \rightarrow 1)$ isotopologues and $\operatorname{HCO}^{+}(J=4 \rightarrow 3)$, but we will only discuss the $\mathrm{CO}$ isotopologues and continuum results in this paper.

\subsection{Spatial Scale Sensitivity}

The incomplete sampling of the uv-plane limits the maximum size of structures that can be fully recovered with an interferometer. Moreover, the inclusion of only B and C or Extended and Compact configurations of CARMA and the SMA, respectively, limits the sensitivity to spatially extended objects. In order to characterize the sensitivity to different spatial scales, we generated a series of uniform brightness model images of symmetric disks having a variety of diameters and sampled them with the uv-coverage of the same configurations as our observations. We find that $50 \%$ of the model flux density can be recovered at $1.3 \mathrm{~mm}$ from a $4^{\prime \prime}$ diameter disk in CARMA C configuration, a 1'75 disk for CARMA B configuration, a $9^{\prime \prime}$ disk for SMA Compact configuration, and a 4 " disk for SMA Extended configuration. The SMA Extended configuration observations at $850 \mu \mathrm{m}$ can recover a 2!7 disk. Note that these sizes are optimistic because the uv-coverage in the simulations is not split between sources as in the observations and some observed data are flagged. Furthermore, a uniform brightness disk is not representative of the structure of protostellar sources, but this example is meant to present a limiting case.

\section{RESULTS}

\subsection{Continuum Emission Structure}

We detect $1.3 \mathrm{~mm}$ dust continuum emission toward all observed sources in both $\mathrm{B}$ and $\mathrm{C}$ configurations. ${ }^{12}$ The detected continuum structures broadly fit into four categories: disk-like structures (Section 3.1.1), candidate multiples (Section 3.1.2), featureless or unresolved (Section 3.1.3), and asymmetric resolved structures (Section 3.1.4). Some of the continuum data are highly structured in the combined B- and $\mathrm{C}$-array imaging, and others may only have resolved structure in the highest-resolution data (B-array). Images of the Class 0 protostars with sensitivity to structure on arcsecond scales with the combined B- and C-array data sets are shown in Figure 1. The highest-resolution images, emphasizing structure on subarcsecond scales, are shown in Figure 2. Furthermore, two Class I protostars were within the primary beams of Class 0 sources, and their images are shown in Figure 3. The $1.3 \mathrm{~mm}$ flux densities, rms noise, and resolution for each image are given in Table 4. In addition, we fit two-dimensional Gaussians to the images shown in Figures 1 and 2. This was done with the imfit task in $\mathrm{CASA}^{13}$ to determine the deconvolved source sizes and position angles; these values are listed in Table 4.

For a source to be classified as "disk-like," it must have a deconvolved position angle within $30^{\circ}$ of orthogonal to the outflow axis; the relative position angles of outflow and continuum sources are given in Table 4. Disks are expected to be aligned orthogonal to the outflow directions, given that the protostar and disk together are thought to be responsible for outflow generation (Frank et al. 2014). We have adopted a somewhat loose limit for the relative position angles for several reasons. First, outflow position angles are generally defined by eye, and asymmetries in the outflow emission can lead to incorrect position angles. Second, outflows can wander with time (e.g., L1157-mm; Gueth et al. 1996) and have bends and kinks (e.g., L1448C; Hirano et al. 2010), adding uncertainty to the outflow axis. Lastly, the continuum emission from the protostellar envelope is entangled with that of the disk, potentially adding systematic uncertainty to the position angle of the continuum.

\subsubsection{Disk-like Structures}

Two sources in our sample show strong evidence of disk-like structures in the dust continuum: L1448 IRS2 (Figures 2 and 4) and Per-emb-14 (Figure 2). They are well resolved in the dust continuum and have deconvolved position angles within $30^{\circ}$ of being orthogonal to the outflow. The outflow directions of these sources are listed in Table 1 and known from both Spitzer IRAC imaging and/or CO outflow mapping.

The disk-like structure toward L1448 IRS2 has a radius of $\sim 300 \mathrm{AU}$ and is at an angle of $71^{\circ}$ with respect to the outflow in the B- and C-array combined image (Figure 1). The SMA Extended and Compact combined image (Figure 4) also shows this structure. The B-array data alone do not appear symmetric, and the emission is only extended toward the southwest at an angle of $68^{\circ}$ with respect to the outflow. This extension does

\footnotetext{
12 Per-emb-24 was only observed in C-array and was not observed in B-array due to only having a $\sim 3 \sigma\left(3.2 \mathrm{mJy}^{\text {beam }}{ }^{-1}\right)$ detection.

13 CASA stands for Common Astronomy Software Application (McMullin et al. 2007) and can be obtained from http://casa.nrao.edu.
} 
Table 3

SMA Observation Log

\begin{tabular}{|c|c|c|c|c|c|c|c|c|}
\hline Sources & Config. & $\begin{array}{l}\text { Date } \\
\text { (UT) }\end{array}$ & $\begin{array}{l}\text { Track Length } \\
\text { (hr) }\end{array}$ & Antennas & $\begin{array}{c}\text { Central Frequencies } \\
(\mathrm{GHz})\end{array}$ & $\begin{array}{l}T_{\text {sys }} \\
(\mathrm{K})\end{array}$ & $\begin{array}{l}\text { Gain Calibrator/Flux Density } \\
\text { (Name, Jy) }\end{array}$ & Flux Calibrator \\
\hline L1448 IRS2 & Compact & 2007 Nov 04 & 10.0 & 7 & 225.434 & 300 & $3 \mathrm{C} 84,3.9$ & Uranus \\
\hline L1448 IRS2 & Compact & 2007 Nov 06 & 10.0 & 7 & 225.434 & 350 & $3 \mathrm{C} 84,3.9$ & Uranus \\
\hline L1448 IRS2 & Extended & 2013 Sep 22 & 9.0 & 5 & $225.434(351.135)$ & $190(450)$ & 3С 84, 0237+288, 10.9, $1.4(9.0,1.3)$ & Uranus \\
\hline L1448 IRS2 & Extended & 2013 Sep 28 & 9.0 & 6 & $225.434(351.135)$ & $200(525)$ & $3 \mathrm{C} 84,0237+288,10.0,1.4(8.8,1.25)$ & Uranus \\
\hline
\end{tabular}

Note. The SMA Extended configuration data were taken in dual-receiver mode. The values in parentheses reflect the data for the $400 \mathrm{GHz}$ receivers. 

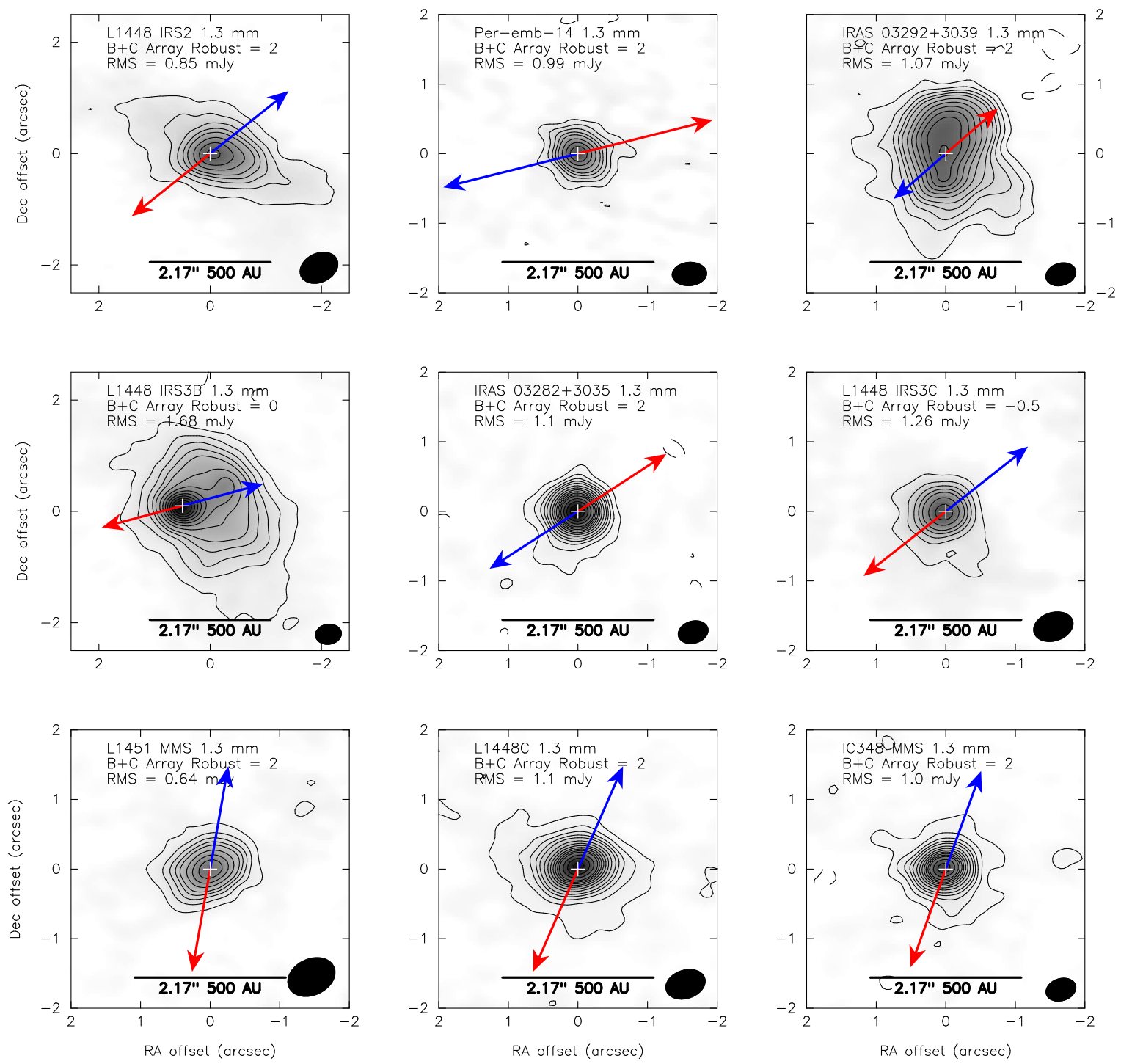

Figure 1. Continuum images at $1.3 \mathrm{~mm}$ of the full sample, emphasizing the structure of the sources on the scale of a few arcseconds. Images are ordered from top to bottom by their amount of resolved structure; the most resolved structures are at the top. The contours in all images are $[-3,3,6,9,12,15,20,25,30,35,40,45,50$, $60, \ldots] \times \sigma$, where $\sigma$ is denoted in each panel and in Table 4 . The beam size is shown in the lower right corner of each image, and exact beam sizes are given in Table 4 . The blue and red arrows denote the direction of the blueshifted and redshifted outflows, respectively.

not appear to trace a discrete source, and the SMA Extended image at $850 \mu \mathrm{m}$ also exhibits this structure (Figure 4).

Per-emb-14, also known as NGC 1333 IRAS 4C (Smith et al. 2000), appears rather featureless in the C- and B-array combined image (Figure 1), but the B-array data alone reveal a compact extended structure at an angle of $70^{\circ}$ with respect to the outflow direction (Figure 2). The radius of this structure is $\sim 100 \mathrm{AU}$ and resembles the expected structure of an edge-on protostellar disk. An outflow has not been detected toward this source in CO (Curtis et al. 2010; Plunkett et al. 2013). However, a fan-shaped scattered light nebula is obvious in Spitzer maps, as well as $\mathrm{H}_{2}$ emission knots extending to the east of the source position from which the outflow position angle is derived; the IRAC imaging is shown in the Appendix. An edge-on orientation could also explain the lack of a $\mathrm{CO}$ outflow detection because the projected velocities would be low and likely confused with the cloud emission.

In addition to Per-emb-14 and L1448 IRS2, the sources L1448C, IRAS 03282+3035, and L1448 IRS3C have deconvolved position angles that are within $30^{\circ}$ of orthogonal to the outflow. Therefore, these sources could also be disk candidates, but they are not as well resolved as Per-emb-14 and L1448 IRS2; they will be discussed further in the following sections.

\subsubsection{Candidate Multiple Sources}

Our high-resolution data also reveal several candidate multiple sources with more than one continuum peak observed. Toward L1448 IRS3B we observe extended structure perpendicular to the outflow in the combined B- and C-array images, as well as the presence of another continuum peak. The B-array imaging alone reveals that there are at least two continuum sources present. The secondary source is located to the northwest by $\sim 0 \prime 9$. The secondary also appears to be marginally resolved, with a separation of $\sim 0.2$, slightly less than the minor axis of the beam $(0,23)$. The nature of the secondary is not immediately clear since it is almost exactly along the axis of the outflow, but it may be a binary companion. The 

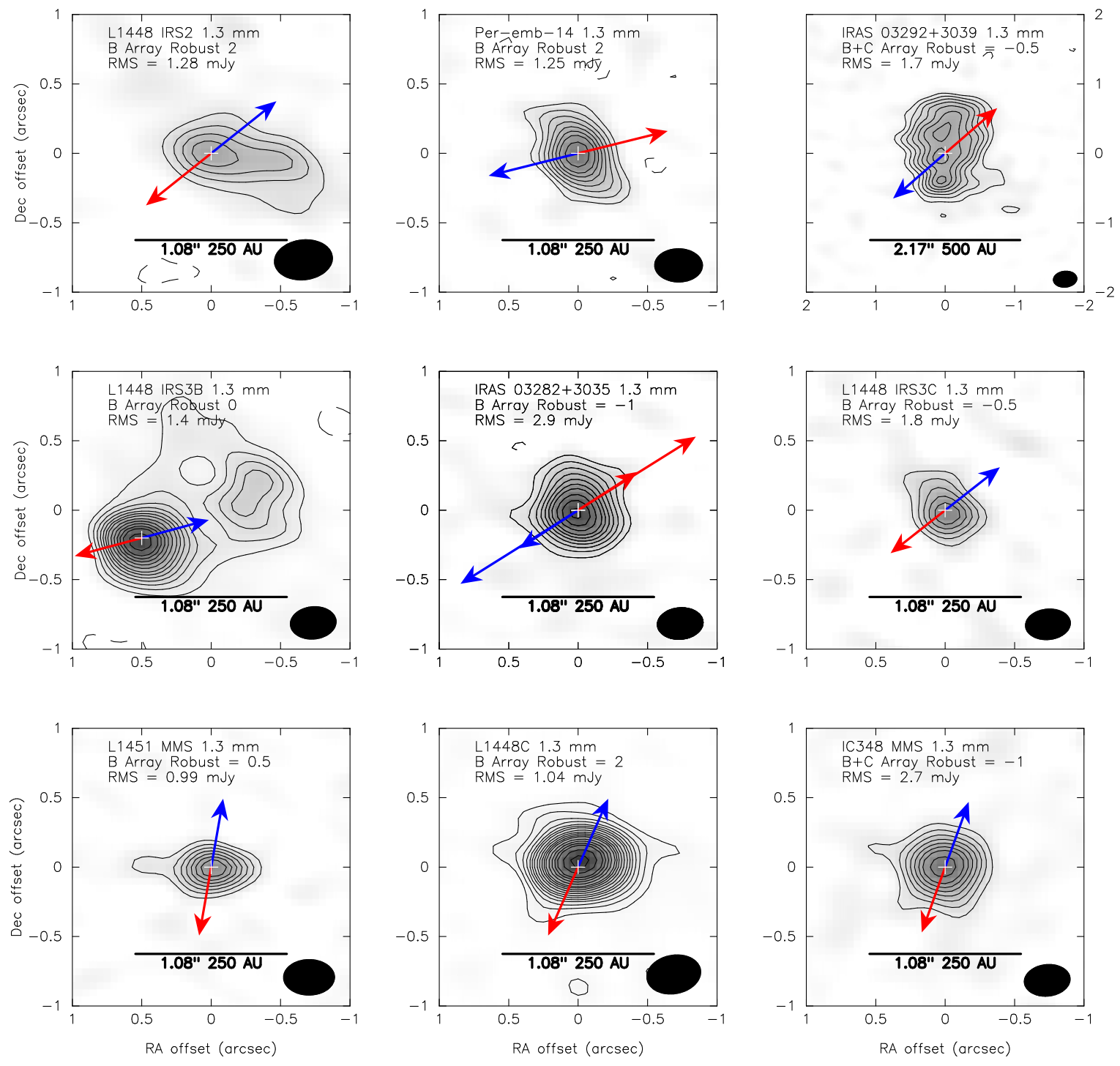

Figure 2. Same as Figure 1, but with emphasis on sub-arcsecond structures. All plots are zoomed in relative to Figure 1, except IRAS $03292+3039$.

deconvolved position angle in Table 4 for the low-resolution image of L1448 IRS3B does not reflect the emission that is extended orthogonal to the outflow because the fitting routine attempts to fit both the main protostar and the companion along the outflow direction.

IRAS 03292+3039 also has structured continuum emission that is extended in the north-south direction at an angle of $49^{\circ}$ with respect to the outflow. There are no strong peaks standing out from this structure, even when imaging with higher resolution, but there are three weak sub-peaks separated from each other by $\sim 0.4$.

\subsubsection{Smooth Continuum Structures}

The sources IC 348-MMS, L1448C, and L1451-MMS have rather smooth continuum structures. They all have resolved envelopes extended over several resolution elements in the B and $\mathrm{C}$ combined imaging as shown in Figure 1. The higherresolution imaging from the B-array data alone does not reveal strong evidence of sub-structure, and they are all consistent with smooth source structure at this resolution (Figure 2). However, the deconvolved position angle of L1448C has an angle of $83^{\circ}$ with respect to the outflow, perhaps indicative of a disk-like structure at the limit of our resolution.

\subsubsection{Asymmetric Resolved Structures}

Several sources show evidence of resolved structure at high resolution, but these are not clearly disk-like in appearance and are often only extended toward one side. L1448 IRS3C (also called L1448 NW; Terebey \& Padgett 1997) appears resolved at an angle of $99^{\circ}$ with respect to outflow, but only toward the northeast (Figure 2). IRAS $03282+3035$ is also extended at a slight angle $\left(109^{\circ}\right)$ with respect to the outflow direction, but only toward the north (Figure 2). The Class I source L1448 IRS3A shows a roughly symmetric structure in the northwest and southeast directions (Figure 3). The outflow direction from this source is uncertain (see Appendix and Kwon et al. 2006), so this could be a disk-like structure, but it is uncertain without clear knowledge of the outflow direction.

The strongest asymmetric structure is found toward IRAS 03292+3039 (Figures 1 and 2). This source was also identified as a candidate multiple, due to its multiple peaks. However, its extended source structure stands out relative to 

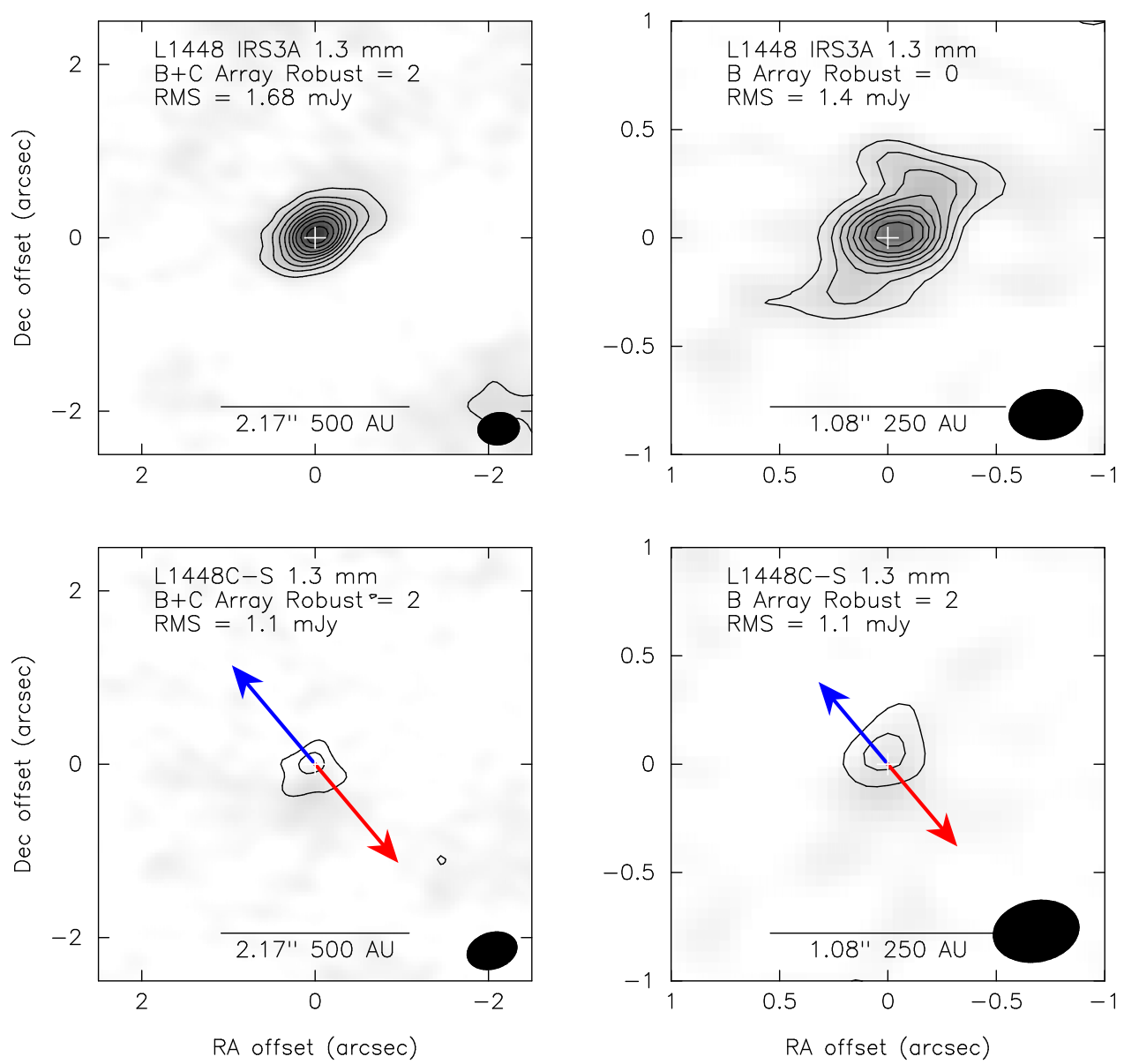

Figure 3. Continuum images at $1.3 \mathrm{~mm}$ of the neighboring Class I sources that were within the primary beam: L1448 IRS3A (top panels) and L1448C-S (bottom panels). The contours in all images are $[-3,3,6,9,12,15,20,25,30,35,40,45,50,60, \ldots] \times \sigma$, where $\sigma$ is denoted in each panel and in Table 4 . The beam size is shown in the lower right corner of each image, and exact dimensions are given in Table 4 . The blue and red arrows denote the direction of the blueshifted and redshifted outflows, respectively.

the other sources in the sample. We will discuss the nature of this source further in Section 5.2.

\subsection{Known Multiple Systems}

Several systems in our sample were previously known to have wide components separated by more than $1^{\prime \prime}$. L1448C is known to have a companion denoted L1448C-S by Jørgensen et al. (2006), also detected by Tobin et al. (2007) and Chen et al. (2013). IC 348-MMS and IRAS 03282+3035 were found to have wide binaries detected at millimeter wavelengths by Chen et al. (2013). Finally, L1448 IRS3 is composed of three components (IRS3B, IRS3A, IRS3C or NW; Looney et al. 2000).

We have confidently detected all components of the L1448 IRS3 system, and we also find that L1448 IRS3B has a closer companion separated by only $0^{\prime} \cdot 9$. We detect the Class I companion toward L1448C with a flux density of $\sim 7 \mathrm{mJy}$, separated by 8"1 ( 1900 AU). However, we do not detect the companion toward IRAS $03282+3035$, reported by Chen et al. (2013) with a separation of 11.5 . We should have detected this source with a signal-to-noise ratio $(\mathrm{S} / \mathrm{N})$ of 7.6. The nondetection of the companion may indicate that it is not real, and it could be a feature extended along the outflow cavity.

The wide binary $\left(\sim 15^{\prime \prime}\right)$ known as IC 348 -MMS2 is not detected because it lies near the edge of the CARMA primary beam, but it does have further detections at both submillimeter and far-infrared wavelengths (Palau et al. 2014). In addition, Rodríguez et al. (2014) detected IC 348-MMS2 and another source at 2 and $3.3 \mathrm{~cm}$ located $\sim 3^{\prime \prime}$ southwest of IC 348-MMS, denoted JVLA 3a, and IC 348-MMS is JVLA 3b. JVLA 3a is undetected in our data, and if it were emitting as a typical protostar, it should produce significant millimeter-wave emission; JVLA 3a is also brighter than IC 348-MMS at $\mathrm{cm}$ wavelengths. The figures shown by Palau et al. (2014) indicate that JVLA 3a may be coincident with the origin of the outflow emission at $4.5 \mu \mathrm{m}$, as well as the Spitzer $24 \mu \mathrm{m}$ source, while IC 348-MMS (JVLA 3b) appears slightly offset.

\subsection{Molecular Line Observations}

We attempted to observe molecular line emission in the course of all the continuum observations, but in nearly all cases the ${ }^{13} \mathrm{CO}$ and $\mathrm{C}^{18} \mathrm{O}$ emission was not strongly detected due to observing three sources per track. Therefore, we obtained follow-up observations of the strong disk candidates L1448 IRS2 and Per-emb-14 with longer integration time in order to characterize their molecular line kinematics in the ${ }^{13} \mathrm{CO}$ and $\mathrm{C}^{18} \mathrm{O}(J=2 \rightarrow 1)$ transitions. The sources in L1448 IRS2, L1448C, L1448 IRS2, and L1451-MMS and the three sources in L1448 IRS3 did have detections of their outflows in ${ }^{12} \mathrm{CO}$ 
Table 4

$1.3 \mathrm{~mm}$ Continuum Properties

\begin{tabular}{|c|c|c|c|c|c|c|c|c|c|c|}
\hline Source & Resolution & $\begin{array}{l}\text { Flux Density } \\
\qquad(\mathrm{mJy})\end{array}$ & $\begin{array}{l}\text { Peak Flux Density } \\
\qquad\left(\mathrm{mJy}_{\text {beam }}^{-1}\right)\end{array}$ & $\begin{array}{c}\text { rms Noise } \\
\left(\mathrm{mJy} \text { beam }^{-1}\right)\end{array}$ & $\begin{array}{l}\text { Beam } \\
\left({ }^{\prime \prime}\right)\end{array}$ & $\begin{array}{c}\text { Beam PA } \\
\left({ }^{\circ}\right)\end{array}$ & $\begin{array}{l}\text { Deconvolved } \\
\text { Size } \\
\left({ }^{\prime \prime}\right)\end{array}$ & $\begin{array}{c}\text { Deconvolved } \\
\text { PA } \\
\left({ }^{\circ}\right)\end{array}$ & $\begin{array}{l}\text { Outflow PA- } \\
\text { Continuum PA } \\
\left(^{\circ}\right)\end{array}$ & $\begin{array}{l}\text { Mass } \\
\left(M_{\odot}\right)\end{array}$ \\
\hline IC 348MMS & low & $163.3 \pm 3.6$ & 75.5 & 1.00 & $0.44 \times 0.32$ & 107.87 & $0.40 \times 0.35$ & $78 \pm 53$ & $72 \pm 53$ & $0.104 \pm 0.002$ \\
\hline $\begin{array}{l}\text { IRAS } 03282 \\
\quad+3035\end{array}$ & low & $191.2 \pm 3.4$ & 99.3 & 1.10 & $0.44 \times 0.32$ & 106.22 & $0.39 \times 0.30$ & $24 \pm 14$ & $101 \pm 14$ & $0.121 \pm 0.002$ \\
\hline L1448C & low & $160.4 \pm 3.7$ & 92.1 & 1.10 & $0.58 \times 0.41$ & 108.02 & $0.45 \times 0.32$ & $81 \pm 19$ & $78 \pm 19$ & $0.102 \pm 0.002$ \\
\hline L1448 IRS2 & low & $77.9 \pm 2.8$ & 26.5 & 0.85 & $0.71 \times 0.52$ & 115.61 & $1.44 \times 0.6$ & $67 \pm 5$ & $71 \pm 5$ & $0.049 \pm 0.002$ \\
\hline L1448 IRS3B & low & $751.8 \pm 10.6$ & 97.5 & 1.68 & $0.47 \times 0.36$ & 97.75 & $1.54 \times 1.34$ & $70 \pm 56$ & $35 \pm 56$ & $0.477 \pm 0.007$ \\
\hline L1448 IRS3C & low & $59.3 \pm 2.9$ & 35.2 & 1.26 & $0.58 \times 0.41$ & 107.76 & $0.35 \times 0.12$ & $23 \pm 11$ & $105 \pm 11$ & $0.038 \pm 0.002$ \\
\hline L1451MMS & low & $27.0 \pm 1.2$ & 22.3 & 0.64 & $0.71 \times 0.52$ & 115.25 & $0.44 \times 0.30$ & $90 \pm 24$ & 90 & $0.017 \pm 0.001$ \\
\hline $\begin{array}{l}\text { IRAS } 03292 \\
\quad+3039\end{array}$ & low & $445.9 \pm 5.6$ & 48.7 & 1.07 & $0.44 \times 0.32$ & 106.88 & $1.2 \times 0.88$ & $176 \pm 7$ & $130 \pm 7$ & $0.283 \pm 0.004$ \\
\hline Per-emb-14 & low & $56.8 \pm 2.3$ & 34.4 & 0.99 & $0.50 \times 0.34$ & 94.14 & $0.45 \times 0.27$ & $31 \pm 13$ & $64 \pm 13$ & $0.036 \pm 0.001$ \\
\hline L1448 IRS3A & low & $74.1 \pm 4.5$ & 39.8 & 1.68 & $0.48 \times 0.37$ & 97.43 & $0.65 \times 0.35$ & $125 \pm 7$ & $30 ? \pm 7$ & $0.047 \pm 0.003$ \\
\hline L1448C-S & low & $6.1 \pm 1.3$ & 7.0 & 1.10 & $0.58 \times 0.41$ & 108.02 & $0.53 \times 0.39$ & $79 \pm 63$ & $141 \pm 63$ & $0.004 \pm 0.001$ \\
\hline IC 348MMS & high & $105.8 \pm 5.8$ & 54.6 & 2.70 & $0.33 \times 0.23$ & 95.95 & $0.32 \times 0.29$ & $22 \pm 82$ & $128 \pm 82$ & $0.067 \pm 0.004$ \\
\hline $\begin{array}{l}\text { IRAS } 03282 \\
\quad+3035\end{array}$ & high & $149.6 \pm 6.7$ & 71.0 & 2.90 & $0.33 \times 0.23$ & 94.79 & $0.36 \times 0.27$ & $16 \pm 29$ & $109 \pm 29$ & $0.095 \pm 0.004$ \\
\hline L1448C & high & $114.4 \pm 3.1$ & 77.7 & 1.04 & $0.39 \times 0.28$ & 100.59 & $0.23 \times 0.16$ & $76 \pm 17$ & $83 \pm 17$ & $0.073 \pm 0.002$ \\
\hline L1448 IRS2 & high & $28.9 \pm 2.6$ & 13.7 & 1.28 & $0.42 \times 0.29$ & 96.42 & $0.86 \times 0.26$ & $70 \pm 6$ & $68 \pm 6$ & $0.018 \pm 0.002$ \\
\hline L1448 IRS3B & high & $152.6 \pm 6.2$ & 54.3 & 1.4 & $0.33 \times 0.23$ & 95.46 & $0.34 \times 0.29$ & $54 \pm 81$ & $51 \pm 81$ & $0.097 \pm 0.004$ \\
\hline L1448 IRS3C & high & $33.3 \pm 3.1$ & 23.5 & 1.80 & $0.33 \times 0.22$ & 95.40 & $0.33 \times 0.06$ & $29 \pm 10$ & $99 \pm 10$ & $0.021 \pm 0.002$ \\
\hline L1451MMS & high & $17.5 \pm 1.5$ & 17.2 & 0.99 & $0.37 \times 0.26$ & 90.33 & point & $\ldots$ & $\ldots$ & $0.011 \pm 0.001$ \\
\hline $\begin{array}{l}\text { IRAS } 03292 \\
\quad+3039\end{array}$ & high & $301.2 \pm 7.6$ & 27.2 & 1.70 & $0.34 \times 0.23$ & 95.58 & $1.1 \times 0.74$ & $175 \pm 7$ & $131 \pm 7$ & $0.191 \pm 0.005$ \\
\hline Per-emb-14 & high & $45.6 \pm 2.6$ & 26.3 & 1.25 & $0.35 \times 0.24$ & 269.46 & $0.40 \times 0.1$ & $25 \pm 8$ & $70 \pm 8$ & $0.029 \pm 0.002$ \\
\hline L1448 IRS3A & high & $55.8 \pm 3.5$ & 29.0 & 1.4 & $0.34 \times 0.23$ & 94.82 & $0.51 \times 0.22$ & $137 \pm 10$ & $18 ? \pm 12$ & $0.035 \pm 0.002$ \\
\hline L1448C-S & high & $7.5 \pm 1.4$ & 6.7 & 1.04 & $0.39 \times 0.28$ & 100.59 & point & $\ldots$ & $\ldots$ & $0.005 \pm 0.001$ \\
\hline
\end{tabular}

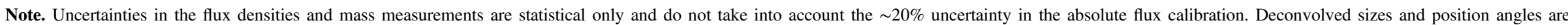
determined using the imfit task in CASA. The error in the relative angle between the deconvolved source position angle and outflow position angle assumes no error in the outflow position angle. 

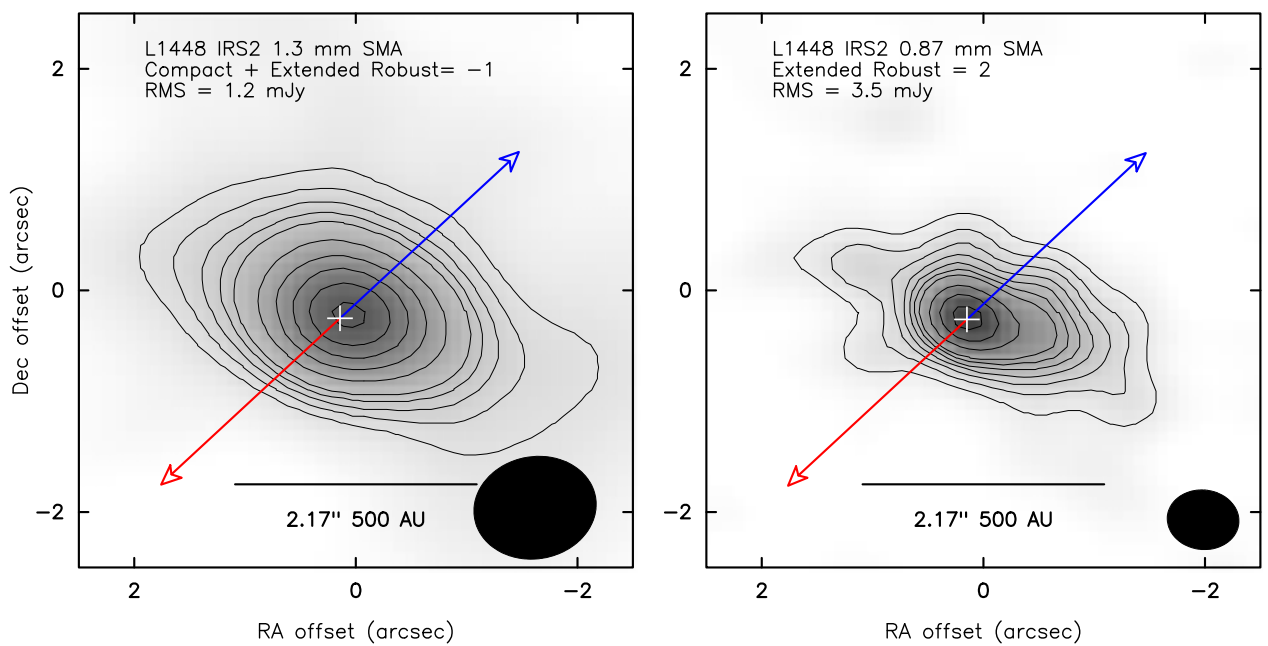

Figure 4. L1448 IRS2 images at $1.3 \mathrm{~mm}$ (Extended and Compact) and $850 \mu \mathrm{m}$ (Extended-only) from the SMA. The $1.3 \mathrm{~mm}$ image traces similar structure as the CARMA $1.3 \mathrm{~mm}$ images in Figures 1 and 2 . The $850 \mu \mathrm{m}$ image at higher resolution still traces larger-scale structure, but does show the asymmetry evident in the higher-resolution $1.3 \mathrm{~mm}$ image. The contours in the $1.3 \mathrm{~mm}$ images start at $7 \sigma$, increasing by $2 \sigma$ until $15 \sigma$, at which point they increase by $5 \sigma$; the $850 \mu \mathrm{m}$ contours increase in the same sense, except that they start at $3 \sigma$. At $1.3 \mathrm{~mm}$ and $850 \mu \mathrm{m} \sigma=1.2$ and $3.5 \mathrm{mJy}$, respectively. The beam sizes are $1^{\prime \prime} 11 \times 0.92$ and $0.65 \times 0.54$ at $1.3 \mathrm{~mm}$ and $850 \mu \mathrm{m}$, respectively. The blue and red arrows denote the direction of the blueshifted and redshifted outflows, respectively.

emission (in addition to ${ }^{13} \mathrm{CO}$ for L1448 IRS2), and we discuss these observations in the Appendix.

\subsubsection{L1448 IRS2}

The presence of the large continuum structure toward L1448 IRS2 is suggestive of a disk, but molecular line observations are necessary to characterize its kinematics and determine its nature. The $\mathrm{C}^{18} \mathrm{O}(J=2 \rightarrow 1)$ integrated intensity maps and position-velocity diagrams are shown in Figure 5. The lowerresolution image (top panels) is a combination of SMA Compact and CARMA C-array observations, showing a velocity gradient on $\sim 5^{\prime \prime}(1200 \mathrm{AU})$ scales in both the blueshifted and redshifted integrated intensity and positionvelocity plot. The position angle between the peak blueshifted and redshifted emission is $70^{\circ}$ east of north, making a $68^{\circ}$ angle with respect to the outflow. Therefore, this velocity gradient could be interpreted as rotation.

The SMA Compact and Extended map is shown in the bottom panel of Figure 5 with the respective position-velocity plot. The addition of the sensitive Extended array data shows that the velocity gradient direction changes to a position angle of $100^{\circ}$ east of north and is now more in the direction of the outflow on $\sim 2^{\prime \prime}$ scales. There are several possibilities that could produce a velocity gradient along the outflow that we will discuss further in Section 5.2. Therefore, we cannot confirm rotation on scales less than $\sim 300$ AU toward L1448 IRS2.

\subsubsection{Per-emb-14}

We have also obtained higher-sensitivity molecular line imaging toward Per-emb-14 (NGC 1333 IRAS 4C) in the $\mathrm{C}^{18} \mathrm{O}$ $(J=2 \rightarrow 1)$ transition with CARMA in $\mathrm{C}$-array; ${ }^{13} \mathrm{CO}$ $(J=2 \rightarrow 1)$ was not detected. The $\mathrm{C}^{18} \mathrm{O}$ emission is weak, but we have made integrated intensity maps of the red- and blueshifted emission toward the source that are shown in Figure 6 . The blue- and redshifted emission are offset from each other, with the velocity gradient being perpendicular to the outflow and consistent with the axis of the resolved continuum source. The offset is only $\sim 0.5$, which is about $40 \%$ of the beam width; however, the velocity centroid accuracy is approximately equivalent to the beam width divided by the $\mathrm{S} / \mathrm{N}$ (Reid et al. 1988), 0 '25 in this case. While the gradient is most likely real, the $\mathrm{S} / \mathrm{N}$ is not high enough to determine if it is indicative of rotational support.

\subsection{Visibility Amplitude Profiles}

The well-sampled uv-plane provided by CARMA produces deconvolved images that reliably recover complex structure; however, the visibility amplitude data themselves still provide details of source structure that are not immediately apparent in the deconvolved images. The circularly averaged visibility amplitudes are plotted against projected uv-distance for each source and are shown in Figure 7, with uv-coverage out to $\sim 600 \mathrm{k} \lambda$. These data show a variety of structures from roughly constant amplitude out to $600 \mathrm{k} \lambda(0.33)$ to very little emission on baselines longer than $\sim 200 \mathrm{k} \lambda$ (scales smaller than $1^{\prime \prime}$ ).

L1448C and L1451-MMS exhibit the flattest visibility amplitudes. L1448C shows a linear decline in the log-log plots; the linearity is evident in L1448C out to the longest baselines. L1451-MMS shows the least amount of visibility amplitude decline of all the observed sources.

Per-emb-14, IRAS 03282+3035, IC 348-MMS, and IRAS 03292+3039 all decrease slowly until a scale specific to each source and then drop quickly. The scales are consistent with the size of the resolved structures apparent in the deconvolved images. L1448 IRS2 also had $\sim 4^{\prime \prime}$ diameter flattened structure, but the visibility amplitudes decrease more slowly than IRAS $03292+3039$, likely due to the structure being smaller in one dimension. L1448 IRS2 also shows variations at uv-distances $>100 \mathrm{k} \lambda$, likely due to the resolved structure seen in the images on $>1^{\prime \prime}$ scales.

L1448 IRS3B showed clear evidence of multiplicity in its deconvolved image (Figure 2 ). The visibility amplitudes flatten at $>100 \mathrm{k} \lambda$ and then begin to drop at $\sim 250 \mathrm{k} \lambda\left(0^{\prime \prime} 8\right)$, the approximate separation of the candidate companion source. The visibility amplitude structures of L1448 IRS3C and L1448 IRS3A are difficult to interpret due to their proximity to L1448 IRS3B; moreover, both sources show abrupt drops in visibility amplitude at $\sim 200$ and $\sim 400 \mathrm{k} \lambda$. The visibility data will be 

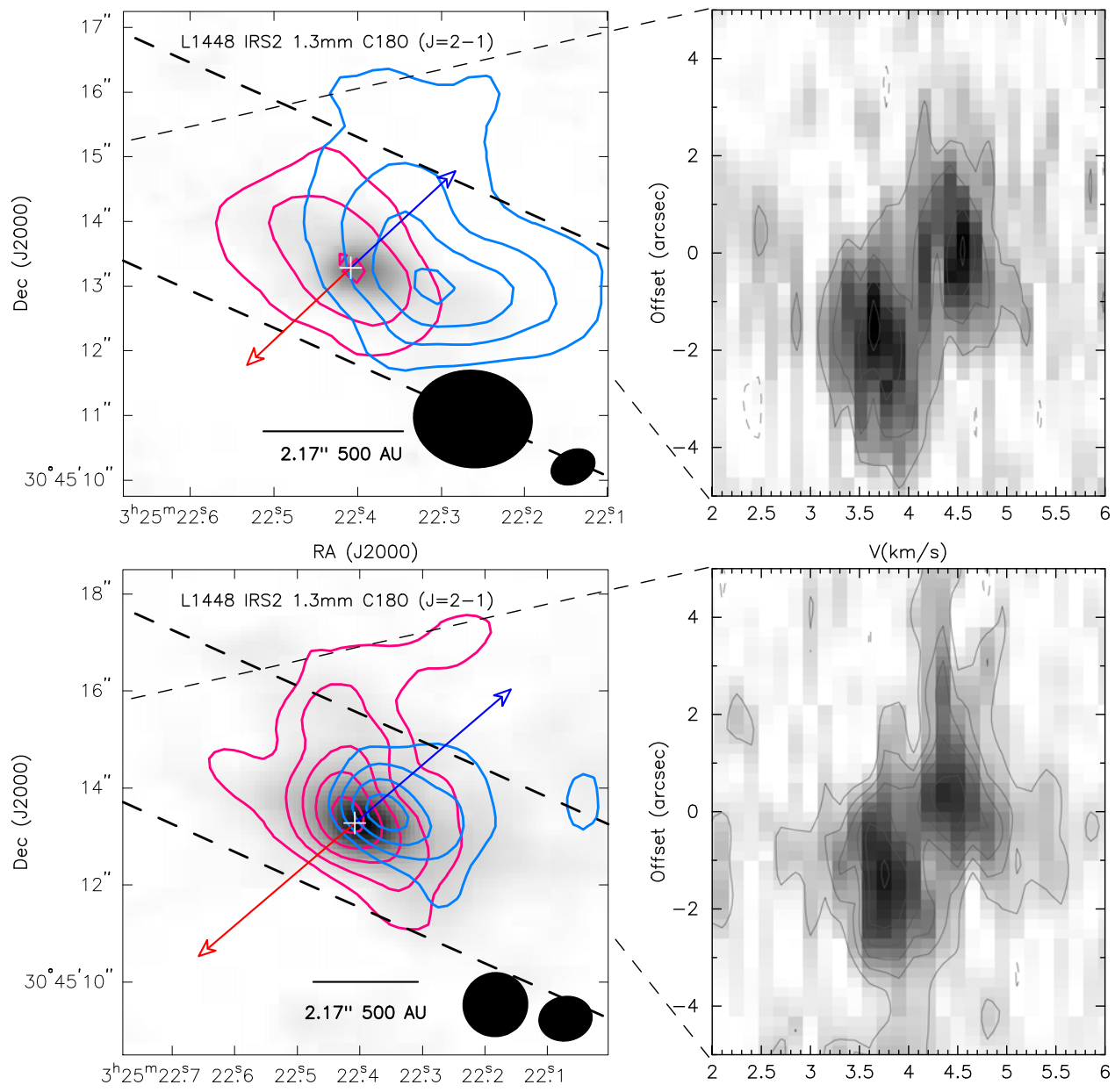

RA (J2000)

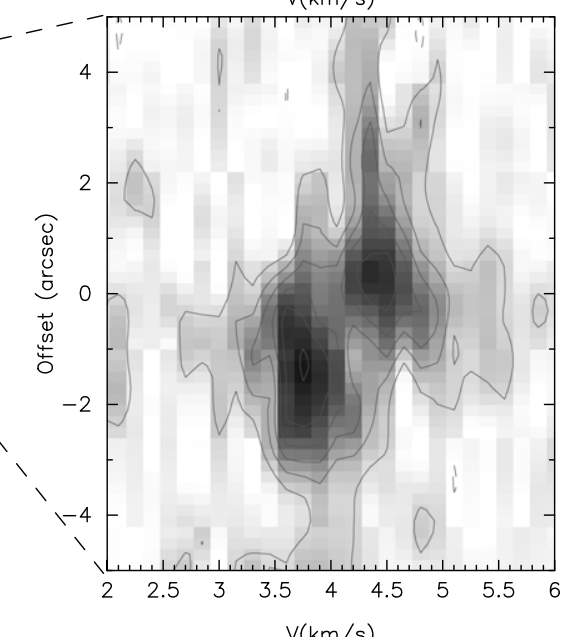

Figure 5. Integrated intensity images of red- and blueshifted $\mathrm{C}^{18} \mathrm{O}(J=2 \rightarrow 1)$ emission overlaid on $1.3 \mathrm{~mm}$ dust continuum imaging and position-velocity diagrams taken from the region enclosed by the parallel dashed lines (right). The top panels are from CARMA C- and B-array and SMA C ${ }^{18} \mathrm{O}$ Compact data, with 0.5 tapering applied; the lower-resolution data show a clear velocity gradient on $5^{\prime \prime}\left(\sim 1200 \mathrm{AU}\right.$ scales) at an angle of $70^{\circ}$ with respect to the outflow position angle. The bottom panels show the higher-resolution SMA Compact and Extended array observations of $\mathrm{C}^{18} \mathrm{O}$; the direction of the velocity gradient changes to have an angle of only $30^{\circ}$ with respect to the outflow position angle on scales less than $2^{\prime \prime}(\sim 500 \mathrm{AU})$. The contours in the CARMA and SMA imaging (top) are $[-6,6,9, \ldots] \times \sigma$, where sigma is 0.46 and $0.48 \mathrm{~K}$ for the red- and blueshifted emission, respectively. The contours for the SMA-only imaging are $[-5,9,13,17] \times \sigma$, where sigma is 0.68 and $0.62 \mathrm{~K}$ for the red- and blueshifted emission, respectively. The larger beams are for the line data and are $1.82 \times 1.48$ and $1.31 \times 1.29$ for the low- and high- resolution images, respectively; the smaller beams are for the continuum data, the same as in Figures 1 and 4 . The blue and red arrows denote the direction of the blueshifted and redshifted outflows, respectively.

further interpreted in conjunction with radiative transfer models in Section 4.

\subsection{Dust Continuum Masses}

The dust emission observed toward the protostars is directly proportional to the mass of the emitting dust, provided that the dust opacity, the temperature of the emitting dust, and the optical depth are known. At millimeter wavelengths the dust opacities are low enough such that optically thin emission is a reasonable assumption. However, the dust opacity is only known to within a factor of 3-5 from models (e.g., Ossenkopf \& Henning 1994; Ormel et al. 2011) and empirical estimates (e.g., Beckwith et al. 1990). Moreover, there can be opacity variations from source to source (Kwon et al. 2009; Chiang et al. 2012) and radial opacity variations in envelopes (Kwon et al. 2009) and disks (Pérez et al. 2012). Since these sources all have protostars, there will also be a temperature gradient in the envelope. However, for simplicity, we will assume a constant, average temperature of $30 \mathrm{~K}$, appropriate for the environs within a few $\times 100 \mathrm{AU}$ from the protostar.

The mass of the emitting dust can be calculated with the relationship

$$
M=\frac{D^{2} F_{\lambda}}{\kappa_{\lambda} B_{\lambda}\left(T_{\text {dust }}\right)}
$$

where $D$ is the distance, $\kappa$ is the dust opacity at the observed wavelength, $B\left(T_{\text {dust }}\right)$ is the Planck function, and $T_{\text {dust }}$ is the assumed temperature of the emitting material. We are adopting the dust opacities of Ossenkopf \& Henning (1994) for this calculation $\left(\kappa_{1.3 \mathrm{~mm}}=0.899 \mathrm{~cm}^{2} \mathrm{~g}^{-1}\right.$, dust-only opacity), which are appropriate for the conditions of protostellar clouds. Finally, the total mass can be calculated by assuming a dustto-gas mass ratio, the standard value of which is $1: 100$. The systematic uncertainty in the derived masses can be factors of several due to uncertainties in the dust opacity and dust temperature. 


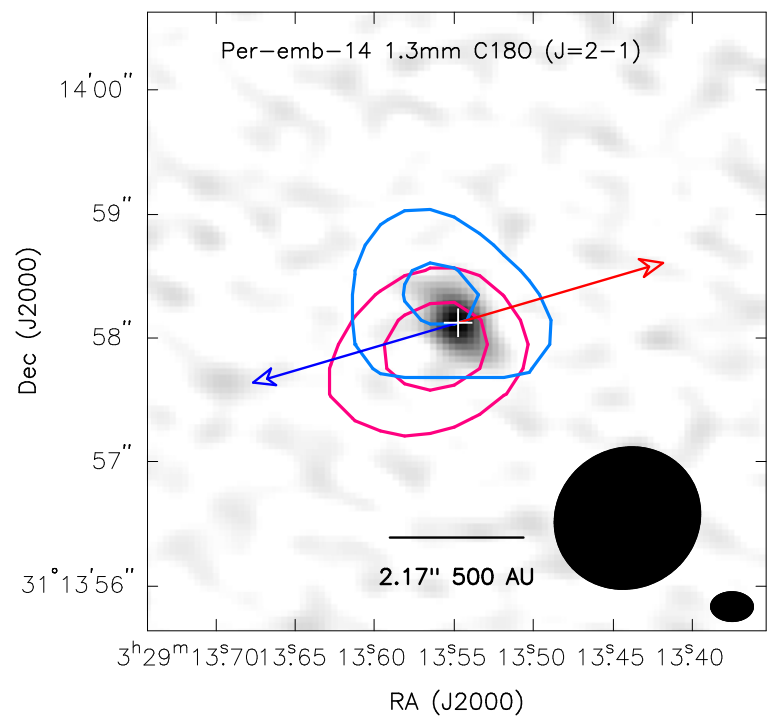

Figure 6. Per-emb-14 (NGC 1333 IRAS4C) with CARMA $\mathrm{C}^{18} \mathrm{O}$ overlaid on the $\mathrm{B}$ - and $\mathrm{C}$-array dust continuum image. The integrated intensity of the redand blueshifted emission is suggestive of rotation on the scale of the disk candidate, but the $\mathrm{S} / \mathrm{N}$ is far too low for us to determine if the rotation is consistent with Keplerian. The contours are $\pm 3 \sigma$ and $5 \sigma$, where $\sigma=1.74$ and $1.35 \mathrm{~K}$ for the red- and blueshifted maps, respectively. The larger beam drawn in the lower right corresponds to the $\mathrm{C}^{18} \mathrm{O}$ data and is $1^{\prime \prime} 19 \times 11^{\prime \prime} 11$; the smaller beam is for the continuum data and is the same as in Figure 2. The blue and red arrows denote the direction of the blueshifted and redshifted outflows, respectively.
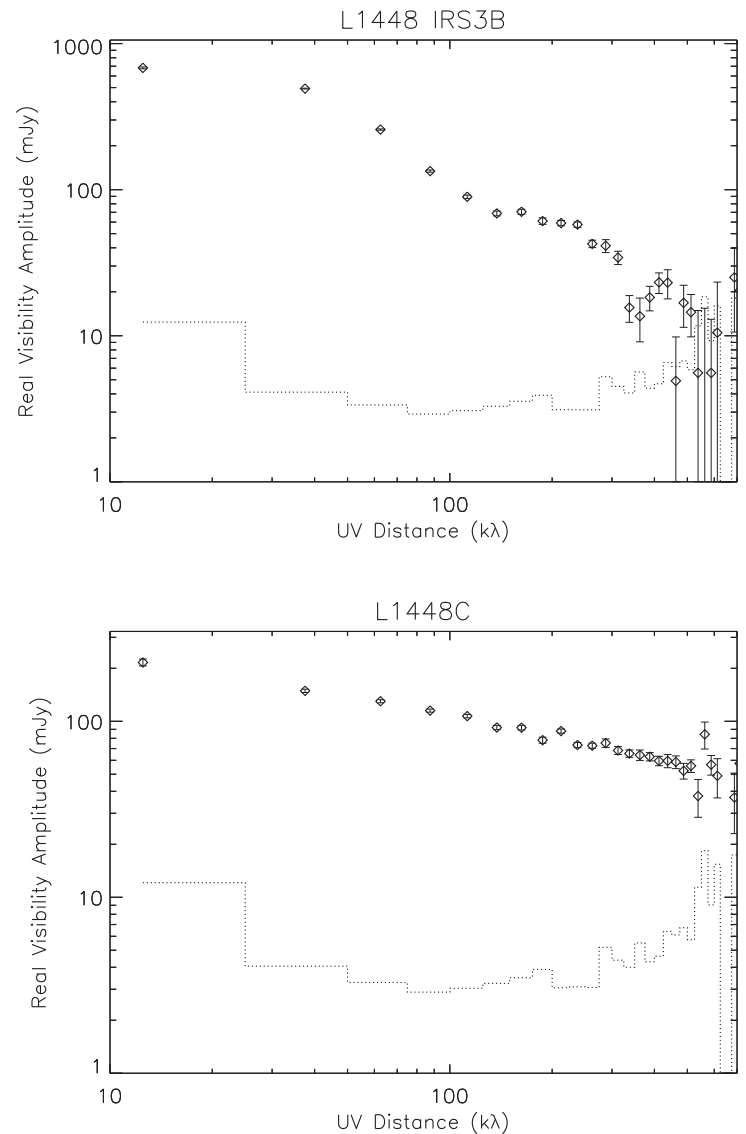

We will use this method to estimate the mass of the emitting material from the observed flux density at $1.3 \mathrm{~mm}$, assuming that we are only detecting thermal dust emission. To measure the flux densities, we will employ two methods. First, we will measure the flux density in the deconvolved images at both low and high resolution and use these flux measurements to calculate the mass. Second, we will measure the flux densities directly from the visibility amplitudes on 50 and $100 \mathrm{k} \lambda$ scales. On these scales the envelope contribution is small and the residual contribution can be estimated from single-dish flux density measurements.

\subsubsection{Masses from Deconvolved Images}

To measure the continuum flux densities directly from the deconvolved images, we sum the flux above the $3 \sigma$ level within a 2.!5 aperture around the protostar position. Thus, we only include the flux density bounded by our $3 \sigma$ image contours shown in Figures 1 and 2. We perform these measurements on both the lower-resolution images and the high-resolution images. The integrated flux densities, peak flux densities, rms noise, and inferred masses are given in Table 4.

The lowest mass measured in the low-resolution imaging is $0.017 M_{\odot}$ toward L1451-MMS, and the largest mass is $0.48 M_{\odot}$ toward L1448 IRS3B. These mass measurements would include contributions from both the disk and envelope. The median and average low-resolution masses are 0.1 and 0.13 $M_{\odot}$, respectively, with a standard deviation of $0.14 M_{\odot}$. The
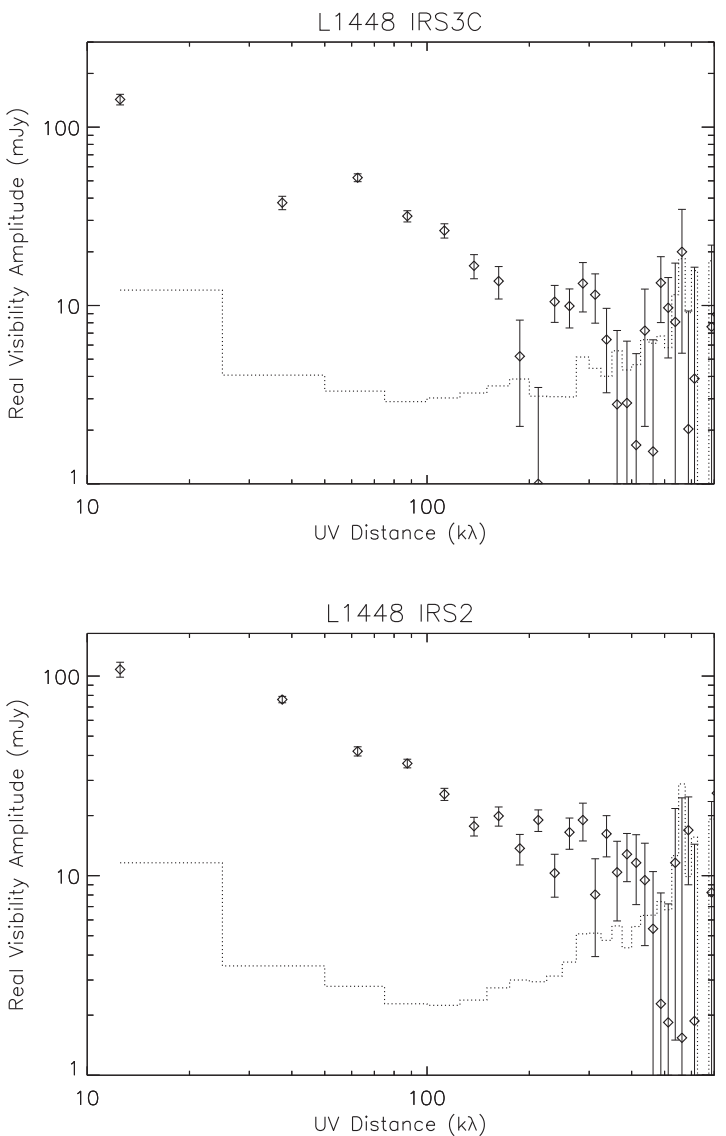

Figure 7. Binned visibility amplitude plots vs. projected uv-distance at $1.3 \mathrm{~mm}$. The visibility data are averaged within $25 \mathrm{k} \lambda$ bins. The sources show a variety of structures from flat visibility amplitudes toward L1451-MMS, power-law decline in L1448C, high scatter for L1448 IRS2 indicating resolved structure, steep declines at a variety of uv-distances (Per-emb-14, IRAS 03292+3039, IRAS 03282+3035), and evidence of multiplicity in L1448 IRS3B. The dotted line is the expected visibility amplitude from noise alone. 
masses from the high-resolution data, on the other hand, have filtered more of the large-scale emission and may better probe the mass from the compact inner envelope and/or disk. The largest high-resolution mass is $0.19 M_{\odot}$ toward IRAS $03292+3039$, and the lowest is $0.011 M_{\odot}$ toward L1451-MMS. The median and average high-resolution masses are 0.067 and $0.064 M_{\odot}$, respectively, with a standard deviation of $0.054 M_{\odot}$.

The masses at both high and low resolutions have variations over an order of magnitude, though the standard deviation of the high-resolution masses is over a factor of two less than the standard deviation at low resolution. Moreover, there are no trends in mass (or flux density) with other protostar properties
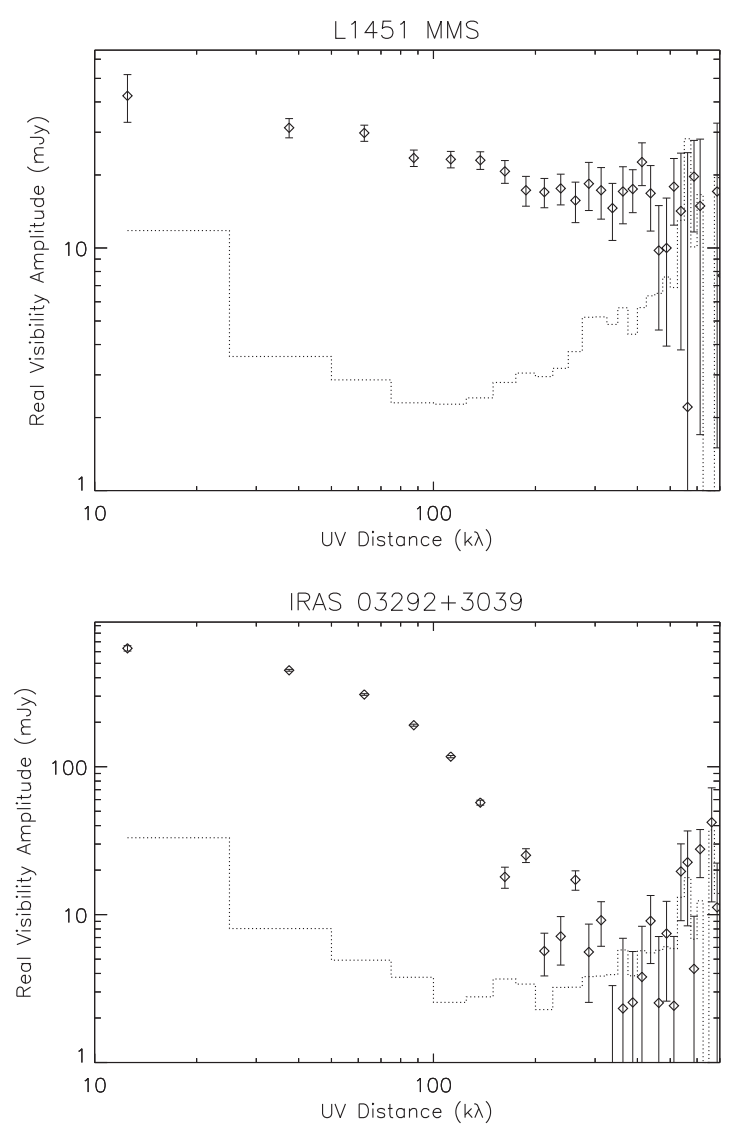

such as bolometric temperature $\left(T_{\mathrm{bol}}\right)$ or bolometric luminosity $\left(L_{\mathrm{bol}}\right)$.

\subsubsection{Masses from Visibility Amplitudes}

We also measured the masses directly from the visibility amplitude data that are shown in Figure 7. This enables us to determine better the spatial scales of the emitting material. We measured the flux densities at 50 and $100 \mathrm{k} \lambda$, corresponding to $\sim 4^{\prime \prime}(920 \mathrm{AU})$ and $\sim 2^{\prime \prime}(420 \mathrm{AU})$, respectively, and any emission must be coming from this or smaller scales. The flux densities and masses are given in Table 5.

The median masses derived from the flux densities at 50 and $100 \mathrm{k} \lambda$ are 0.09 and $0.07 M_{\odot}$, respectively, with respective
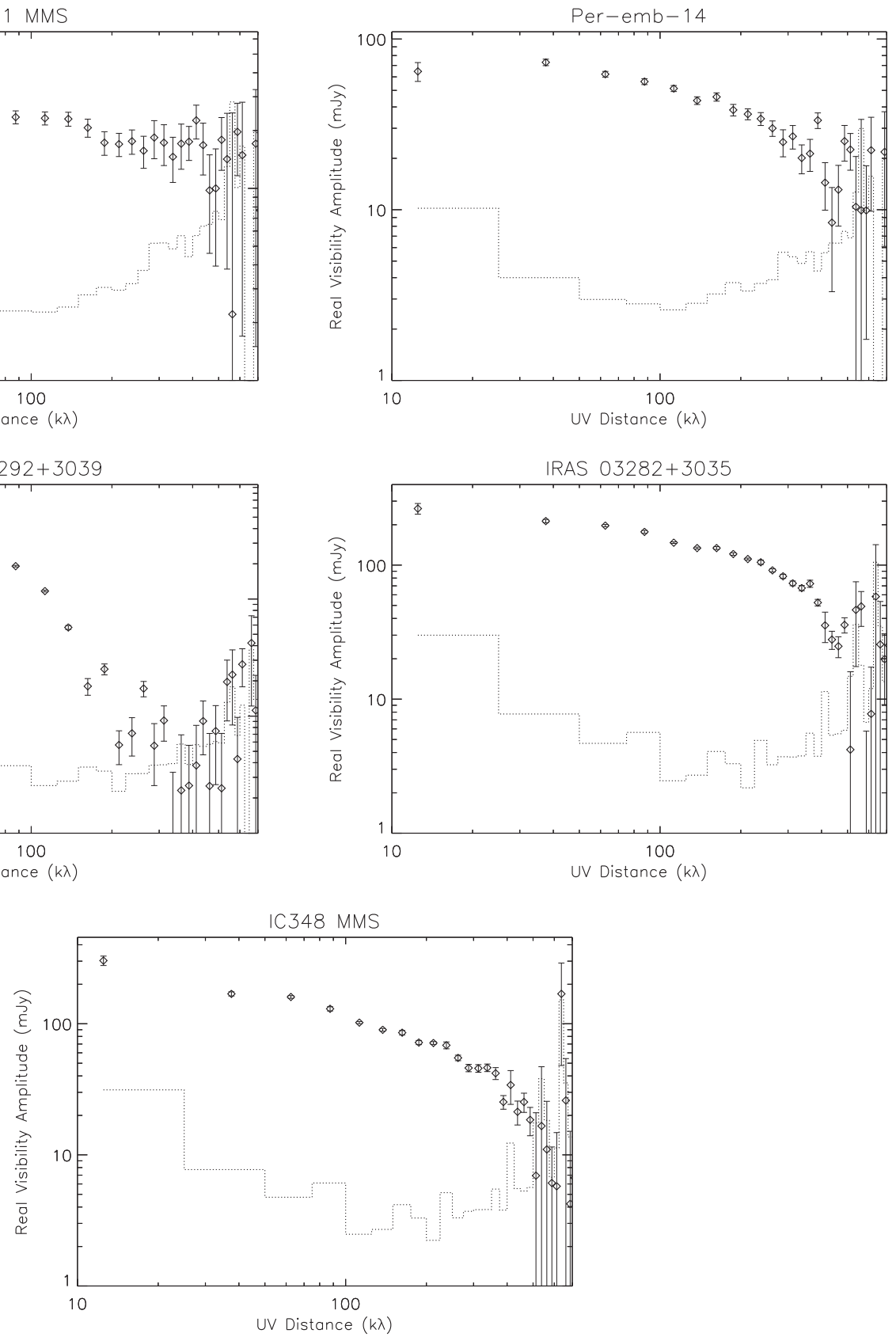

Figure 7. (Continued.) 
average masses of 0.1 and $0.05 M_{\odot}$. The standard deviations of the visibility amplitude masses are lower than those measured from the deconvolved images, 0.08 and $0.03 M_{\odot}$ in units for 50 and $100 \mathrm{k} \lambda$, respectively.

Despite the isolation of emission from small spatial scales, these masses still include any envelope flux that originates on these scales. Jørgensen et al. (2009) developed a method to correct for the residual envelope flux density at $50 \mathrm{k} \lambda$. They determined that an envelope with a $\rho \propto R^{-1.5}$ density profile will contribute at most $4 \%$ of its total emission at $50 \mathrm{k} \lambda$; we verified this and also determined that at $100 \mathrm{k} \lambda$ the envelope contribution is only $2 \%$ of its total flux density. If the density profile were steeper, $\rho \propto R^{-1.8}$ for instance, Jørgensen et al. (2009) found that the envelope could then contribute up to $8 \%$ to the flux density at $50 \mathrm{k} \lambda$.

The total envelope flux density for the sample in Jørgensen et al. (2009) was determined from SCUBA $850 \mu \mathrm{m}$ data. For our sources, there were $1.1 \mathrm{~mm}$ Bolocam data taken toward all protostars in the Perseus cloud. We scaled the $1.1 \mathrm{~mm}$ flux densities to $1.3 \mathrm{~mm}$, assuming $\beta=1.78$ from Ossenkopf \& Henning (1994); the flux densities at $50 \mathrm{k} \lambda$ are typically $10 \%$ to $25 \%$ of the Bolocam flux densities. We then subtracted the estimated envelope contribution of $4 \%$ and $2 \%$ of the scaled Bolocam flux density for 50 and $100 \mathrm{k} \lambda$ scales, respectively.

As an independent check, we applied this method to the resolved disk of L1527 IRS (Tobin et al. 2013). The $870 \mu \mathrm{m}$ and $3.4 \mathrm{~mm}$ visibility amplitudes at $50 \mathrm{k} \lambda$ were 314 and $19.7 \mathrm{mJy}$, respectively. The single-dish flux densities of L1527 at $870 \mu \mathrm{m}$ and $3.3 \mathrm{~mm}$ are 1690 and $33.3 \mathrm{mJy}$, respectively (Shirley et al. 2011). Assuming that the single-dish flux densities only contribute 0.04 of the $50 \mathrm{k} \lambda$ flux, the corrected $50 \mathrm{k} \lambda$ flux densities are 246 and $18.4 \mathrm{mJy}$ at $850 \mu \mathrm{m}$ and $3.4 \mathrm{~mm}$, respectively. These values are close to the integrated flux densities from the highest-resolution images of the resolved disk in Tobin et al. (2013).

The median envelope-corrected masses at 50 and $100 \mathrm{k} \lambda$ are 0.052 and $0.046 M_{\odot}$, respectively, with respective average masses of 0.068 and $0.040 M_{\odot}$. The standard deviations of the masses are 0.08 and $0.03 M_{\odot}$ at 50 and $100 \mathrm{k} \lambda$, respectively, with roughly the same amount of scatter as in Jørgensen et al. (2009). A histogram of disk masses at 50 and $100 \mathrm{k} \lambda$ is shown in Figure 8. We show the corrected and uncorrected masses at 50 and $100 \mathrm{k} \lambda$ versus $T_{\text {bol }}$ in Figure 9. Our sample shows no sign of a correlation between the calculated masses and $T_{\mathrm{bol}}$, while the results of Jørgensen et al. (2009) may have shown a weak correlation. However, the $T_{\text {bol }}$ values we use are calculated with the inclusion of longer-wavelength Herschel data (Sadavoy et al. 2014) and may be more accurate. For instance, $T_{\mathrm{bol}}=47 \mathrm{~K}$ for L1448C in Sadavoy et al. (2014), but Jørgensen et al. (2009) list $T_{\text {bol }}=77 \mathrm{~K}$ for this source and Enoch et al. (2009) give $T_{\text {bol }}=69 \mathrm{~K}$. We also only cover a narrow range of $T_{\text {bol }}$ since we are focused on Class 0 sources, while Jørgensen et al. (2009) had equal numbers of Class 0 and Class I sources.

\section{MODEL COMPARISON}

The interpretation of the $1.3 \mathrm{~mm}$ continuum emission can be aided by comparison to radiative transfer models of axisymmetric envelopes, disks, and envelopes with embedded disks. We have run a small grid of models using the Hyperion code (Robitaille 2011). This is a Monte Carlo radiative transfer code that calculates radiative equilibrium and generates high-S/N images of dust emission using ray tracing. With these models, we aim to determine if the data are consistent with envelopeonly models or if a compact structure is required. We ran three varieties of models: disk-only models, rotating-collapsing envelopes (CMU envelopes; Ulrich 1976; Cassen \& Moosman 1981), and power-law envelopes; both types of envelope models are run with and without disk components.

The models are run with three variations of disk mass 0.0 , 0.01 , and $0.1 M_{\odot}$ and with disk radii of $10,30,50,100,300$, and $500 \mathrm{AU}$. The disks have a surface density profile $\propto R^{-1}$ and a scale height of $10 \mathrm{AU}$ at a radius of $100 \mathrm{AU}$; the surface density profile and scale height are chosen to be consistent with a viscous accretion disk in vertical hydrostatic equilibrium (Shakura \& Syunyaev 1973). The envelopes all have masses of $5.25 M_{\odot}$, a $1 L_{\odot}$ central star, an outer radius of 10,000 AU, and an outflow cavity with a half-opening angle of $10^{\circ}$. The envelope mass corresponds to a CMU model with a mass infall rate of $5 \times 10^{-5} M_{\odot} \mathrm{yr}^{-1}$, and this was chosen to be representative of a young protostellar system dominated by the mass of the envelope. The inner radius of the envelope and disk are equivalent to the dust destruction radiis, adopted to be where the dust temperature is $1600 \mathrm{~K}$. For the CMU envelopes, we assumed that the disk radius was equivalent to the centrifugal radius $\left(R_{C}\right)$, the radius where material is rotationally
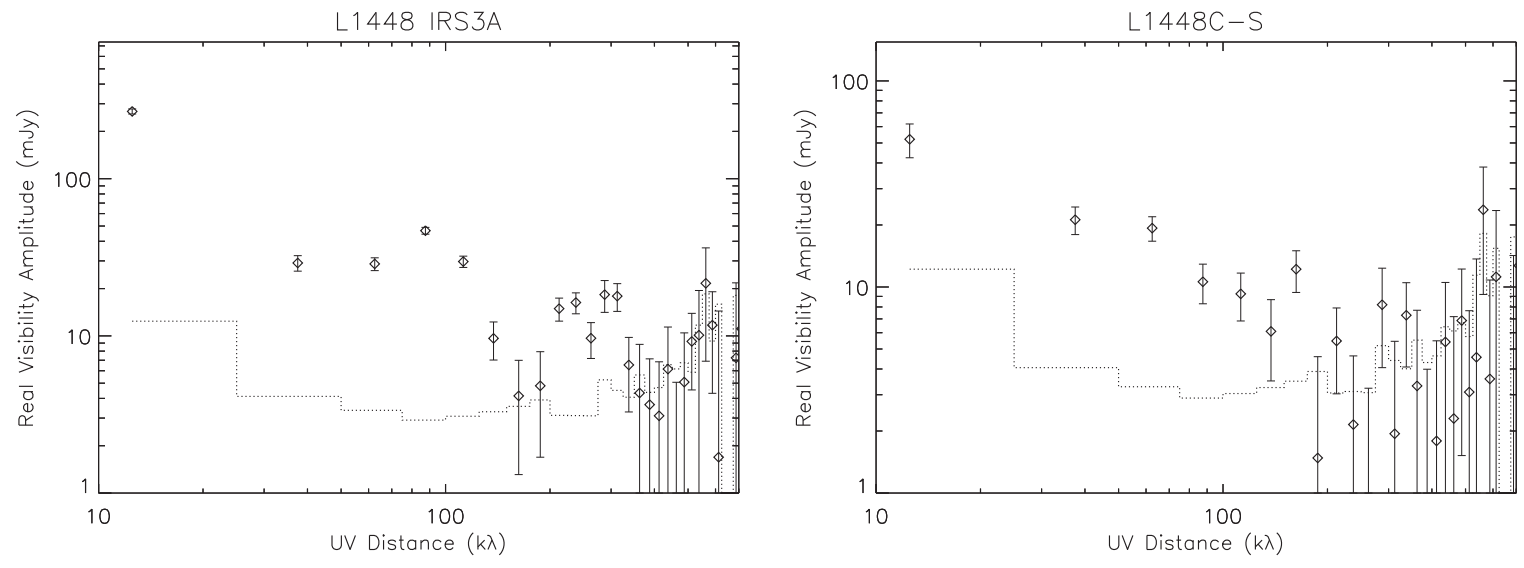

Figure 7. (Continued.) 
Table 5

$1.3 \mathrm{~mm}$ Continuum at 100 and $50 \mathrm{k} \lambda$

\begin{tabular}{|c|c|c|c|c|c|}
\hline Source & $\begin{array}{c}100 \mathrm{k} \lambda \text { Flux Density } \\
(\mathrm{mJy})\end{array}$ & $\begin{array}{c}100 \mathrm{k} \lambda \text { Mass } \\
\left(M_{\odot}\right)\end{array}$ & $\begin{array}{c}50 \mathrm{k} \lambda \text { Flux Density } \\
(\mathrm{mJy})\end{array}$ & $\begin{array}{c}50 \mathrm{k} \lambda \text { Mass } \\
\left(M_{\odot}\right)\end{array}$ & $\begin{array}{c}\text { Bolocam Flux } \\
\text { (mJy) }\end{array}$ \\
\hline IRAS 03282 & $162.0 \pm 3.5$ & $0.103(0.098) \pm 0.002$ & $205.5 \pm 5.1$ & $0.130(0.121) \pm 0.003$ & $540 \pm 20$ \\
\hline IRAS 03292 & $156.5 \pm 2.6$ & $0.099(0.080) \pm 0.002$ & $379.5 \pm 5.3$ & $0.241(0.207) \pm 0.003$ & $1650 \pm 40$ \\
\hline L1448C & $111.0 \pm 2.4$ & $0.070(0.053) \pm 0.002$ & $139.5 \pm 2.9$ & $0.088(0.054) \pm 0.002$ & $1450 \pm 40$ \\
\hline L1448 IRS3A & $38.4 \pm 2.4$ & $0.024(0.018) \pm 0.002$ & $56.2 \pm 3.0$ & $0.036(0.022) \pm 0.002$ & $560^{\mathrm{b}} \pm 70$ \\
\hline L1448 IRS3B & $112.3 \pm 2.4$ & $0.071(0.047) \pm 0.002$ & $378.5 \pm 3.0$ & $0.240(0.197) \pm 0.002$ & $2010^{\mathrm{b}} \pm 70$ \\
\hline L1448 IRS2 & $31.1 \pm 1.8$ & $0.020(0.001) \pm 0.001$ & $59.2 \pm 2.5$ & $0.038(0.0004) \pm 0.002$ & $1460 \pm 50$ \\
\hline L1451MMS & $18.4 \pm 1.7$ & $0.012(0.011) \pm 0.001$ & $24.8 \pm 1.4$ & $0.016(0.014) \pm 0.001$ & $110 \pm 10$ \\
\hline Per-emb-14 & $54.6 \pm 2.2$ & $0.035(0.033) \pm 0.001$ & $67.9 \pm 2.8$ & $0.043(0.041) \pm 0.002$ & $150^{\mathrm{b}} \pm 70$ \\
\hline
\end{tabular}

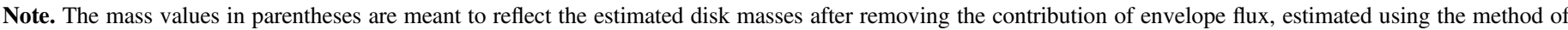

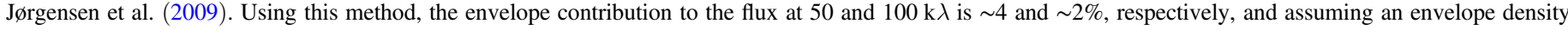

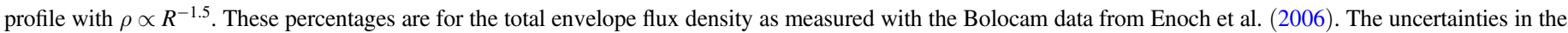
flux densities and mass measurements are statistical only and do not take into account the $\sim 20 \%$ uncertainty in the absolute flux calibration.

${ }^{a}$ Bolocam fluxes are scaled by $(1.1 \mathrm{~mm} / 1.3 \mathrm{~mm})^{\beta}=0.71$, assuming $\beta=1.78$ (Ossenkopf $\&$ Henning 1994), to better match the observed wavelength.

${ }^{\mathrm{b}}$ Estimated flux densities due to blended core.

supported. The power-law envelopes have either a $\rho \propto R^{-1.5}$ or $R^{-2}$ spherical density profile; these density profiles are representative of free-fall collapse and the singular isothermal sphere (Shu 1977) (or, alternatively, the Larson-Penston solution; Larson 1969), respectively.

The models were set up to generate output images at $1.3 \mathrm{~mm}$ with $5 \mathrm{AU}$ pixels and, we Fourier transformed the images using the MIRIAD task fft. We azimuthally averaged the Fourier transformed images to compare with the observed visibility amplitudes. The normalized visibility amplitudes from the envelope-only models $\left(0.0 M_{\odot}\right.$ disks $)$ and the disk-only models are shown in Figure 10. As expected, the CMU models with increasing $R_{C}$ have more rapidly declining visibility amplitudes and a power-law envelope with $\rho \propto R^{-2}$ has a more slowly decreasing visibility amplitude than the $R^{-1.5}$ envelope and CMU envelopes. The disk-only models have flat amplitudes for disks with small radii, but disks with radii $>100 \mathrm{AU}$ show a factor of 5 decline at uv distances $>100 \mathrm{k} \lambda$, and a $100 \mathrm{AU}$ disk has a factor of 5 decline at $500 \mathrm{k} \lambda$. The disk-only models also show variation in visibility amplitudes due to the disks with smaller radii being optically thick, resulting in less emergent flux.

Disks embedded within envelopes are shown in Figures 11 and 12. The addition of the disk components does add some flattening to the visibility amplitude profiles, but they are always declining. The model with $R_{C}=500 \mathrm{AU}$ does, however, have a small upward bump. The disks also change the ratio of the visibility amplitudes at small uv-distances relative to large uv-distances (large scales to small scales, respectively). For assumed envelope masses of $5.25 M_{\odot}$, a 0.01 $M_{\odot}$ disk does not dramatically affect the visibility amplitude profiles, but a $0.1 M_{\odot}$ disk has a more profound effect. We also notice that a $100 \mathrm{AU}, 0.1 M_{\odot}$ disk has a flatter visibility amplitude profile between 100 and $400 \mathrm{k} \lambda$ than disks with radii between 10 and $50 \mathrm{AU}$. This is due to the small, massive disks being optically thick as mentioned in the previous paragraph.
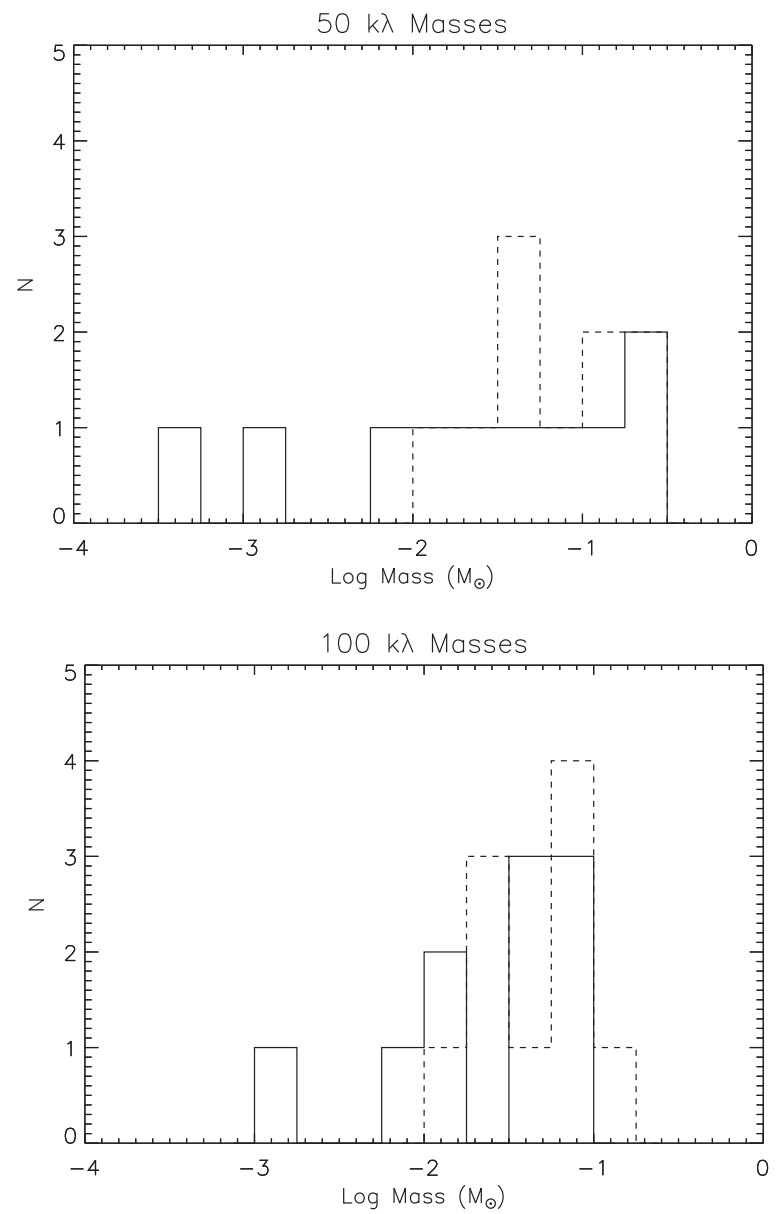

Figure 8. Histogram plot of disk masses inferred from the flux densities at 50 (top) and $100 \mathrm{k} \lambda$ (bottom). Masses that have been corrected for estimated envelope contribution (solid line) and with no correction (dashed line) are shown. The median disk masses corrected for envelope emission are 0.052 and $0.046 M_{\odot}$ at 50 and $100 \mathrm{k} \lambda$, respectively. The respective median disk masses that are not corrected for envelope emission are 0.09 and $0.07 M_{\odot}$, respectively. 
A qualitative comparison of the model visibility amplitude profiles to the data reveals that many sources have profiles that decline more slowly than the models of envelopes with embedded disks. Furthermore, some sources look more like the disk-only models than disk+envelope models (e.g., Peremb-14, IRAS 03282+3035, IC 348-MMS).

A more quantitative comparison of the models and observations is shown in Figure 13, where we plot the ratio of visibility amplitude at $25 \mathrm{k} \lambda$ to the visibility amplitude at $250 \mathrm{k} \lambda$. This plot gives a sense of the relative contribution of the envelope and compact component (modeled as a disk) on spatial scales that are an order of magnitude different, $10^{\prime \prime}(\sim 2300 \mathrm{AU})$ and $1^{\prime \prime}(\sim 230 \mathrm{AU})$ scales. These scales are chosen for both physical and practical reasons; $25 \mathrm{k} \lambda$ is the minimum scale for which we could measure accurate visibility amplitudes due to our chosen array configurations. Furthermore, a $10^{\prime \prime}(\sim 2300 \mathrm{AU})$ scale is likely to be directly associated with gravitational collapse and formation of the protostar, while a disk is expected to form on scales of about $1^{\prime \prime}(\sim 230 \mathrm{AU})$ or smaller. Lastly, at uv-distances greater than $250 \mathrm{k} \lambda$, not all sources had sufficient $\mathrm{S} / \mathrm{N}$ to accurately measure their visibility amplitude.

The disk-only models are shown as plus signs in Figure 13, with an increasing ratio as the disk radius increases, and the power-law envelopes without disks are plotted as the large symbols. We also show the models with increasing disk radii within a given envelope (triangles and squares). An envelope with a $10 \mathrm{AU}, 0.1 M_{\odot}$ embedded disk has large ratios because the disk is optically thick, and the ratios decrease with disk radius until $R=100 \mathrm{AU}$ and then the ratios begin to increase again as the disk itself becomes resolved. These tracks with disk radii can be raised or lowered by changing the ratio of disk mass to envelope mass.

The key result shown in Figure 13 is that for a 25 -to- $250 \mathrm{k} \lambda$ ratio less than $\sim 8$ (the ratio of the $\rho \propto R^{-2}$ envelope, the large diamond in Figure 13), a compact component of some size and mass is required to explain the visibility amplitudes. The most likely form of such a compact component is a protostellar disk. This criterion is fulfilled for six out of nine Class 0 protostars: L1451-MMS, Per-emb-14, L1448 IRS2, L1448C, IC 348MMS, and IRAS 03282+3035. Furthermore, Figure 13 also demonstrates that massive $\left(\sim 0.1 M_{\odot}\right)$, embedded disks with radii $<30 \mathrm{AU}$ may not be apparent toward protostars at $1.3 \mathrm{~mm}$ because the 25 -to- $250 \mathrm{k} \lambda$ visibility amplitude ratio is not significantly lower than the envelope-only case. This happens because emission from the small, massive disks is optically thick, masking much of the emitting material.

\section{DISCUSSION}

The results presented here are currently the largest collection of high-resolution $1.3 \mathrm{~mm}$ continuum observations of Class 0 protostars in a single star-forming region. Moreover, our data
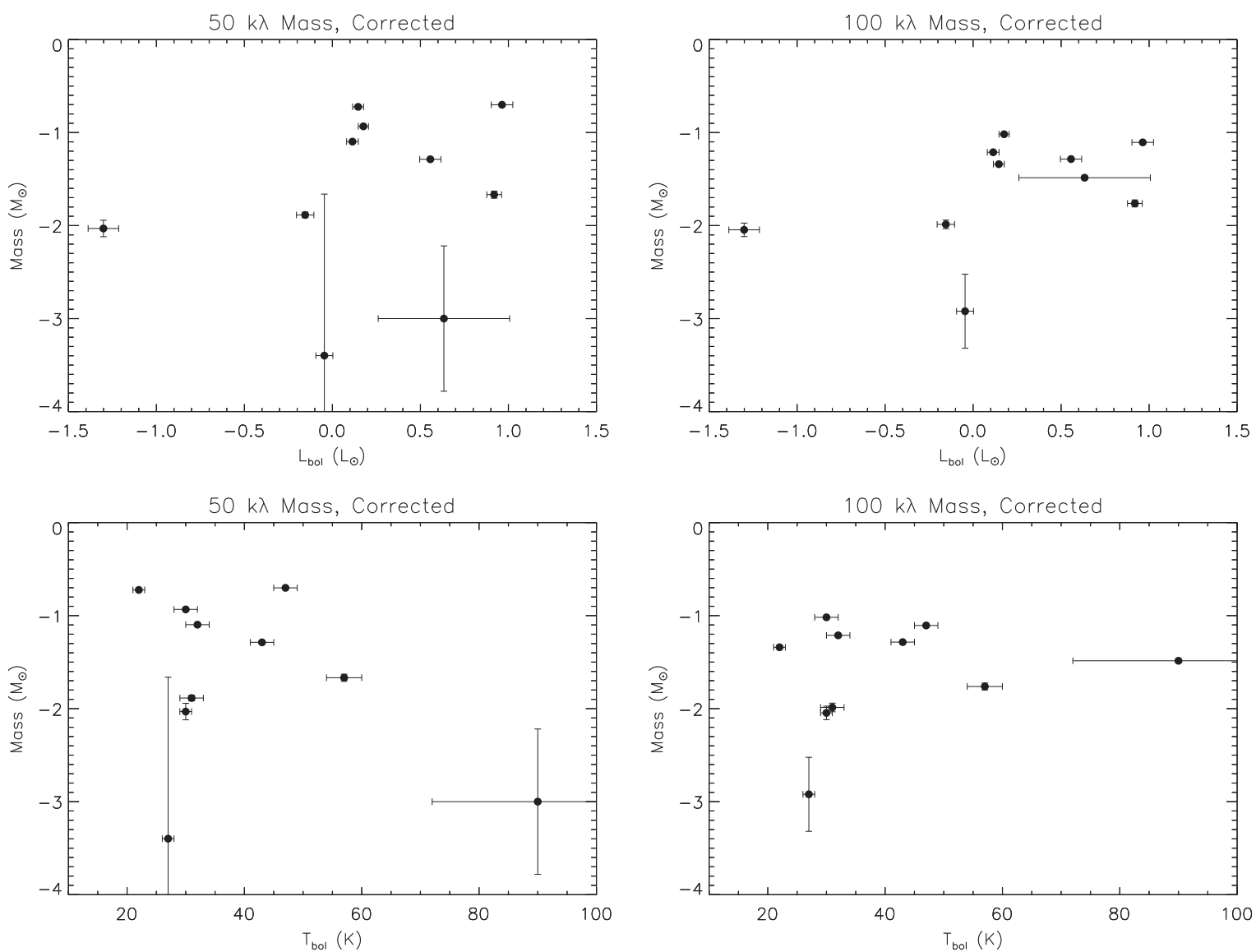

Figure 9. Plot of (corrected) $50 \mathrm{k} \lambda$ disk masses vs. $L_{\mathrm{bol}}$ (top left) and $T_{\mathrm{bol}}$ (bottom left); the same plots but for $100 \mathrm{k} \lambda$ masses are plotted on the right. There is no apparent trend in the inferred disk masses as a function of $L_{\mathrm{bol}}$ or $T_{\mathrm{bol}}$. Jørgensen et al. (2009) also found inconclusive evidence for Class 0 disk masses to trend with these parameters. The lack of correlation indicates that the disk properties (at least mass) may not depend specifically on evolutionary state and luminosity, but rather initial conditions. 

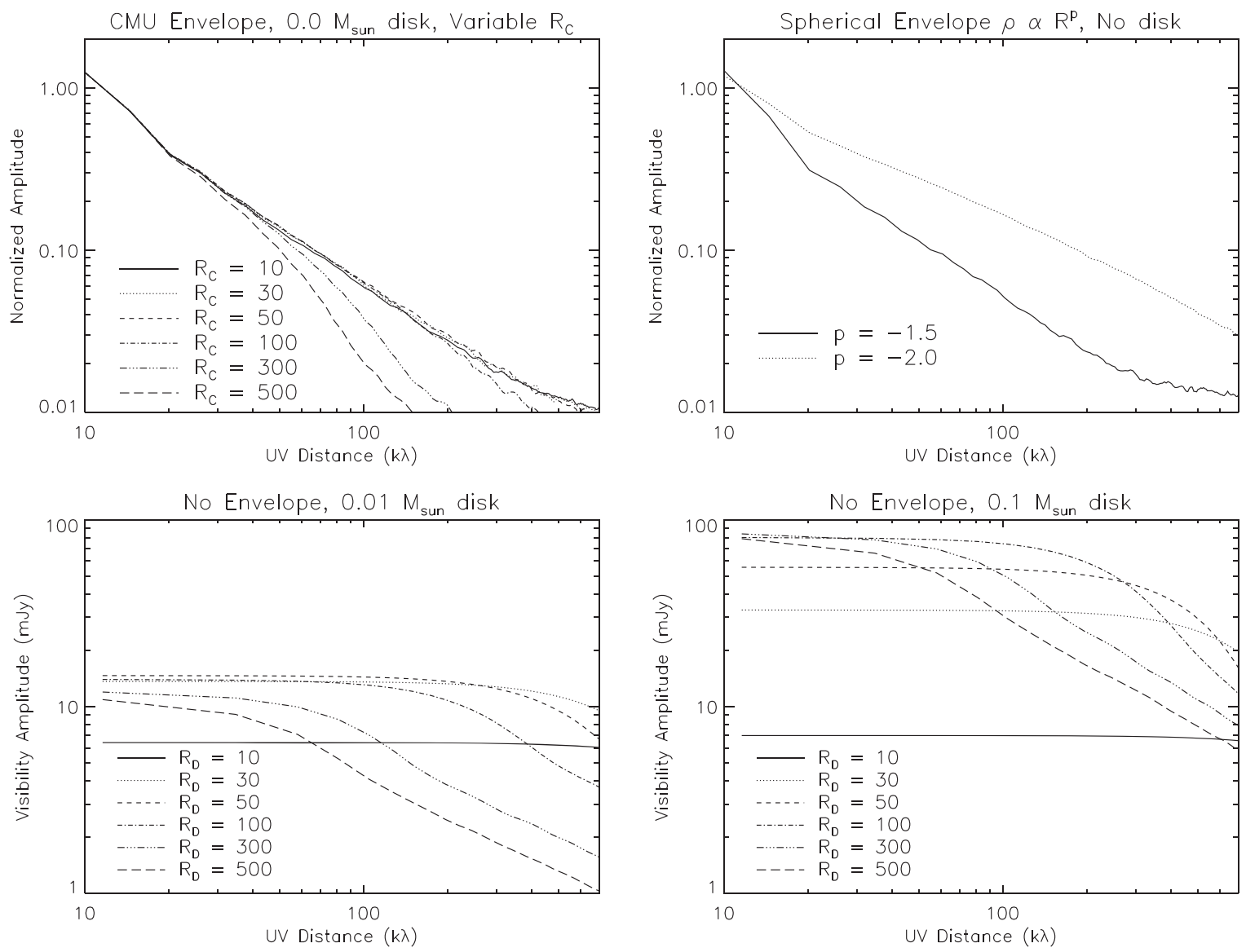

Figure 10. Visibility amplitudes for envelope models with and without disk components. The upper left shows a standard rotating collapse model (CMU) without a disk component. The upper right shows power-law envelopes with density profiles proportional to $R^{-1.5}$ and $R^{-2.0}$ with no embedded disk. The lower left shows diskonly models with sizes between 10 and $500 \mathrm{AU}$ with a total mass of $0.01 M_{\odot}$. The lower right shows disk-only models having a total mass of $0.1 M_{\odot}$. The visibility amplitudes of the disk-only models are not normalized in order to show that for fixed mass, the disks with small radii have high optical depths, thereby reducing the emergent $1.3 \mathrm{~mm}$ flux.
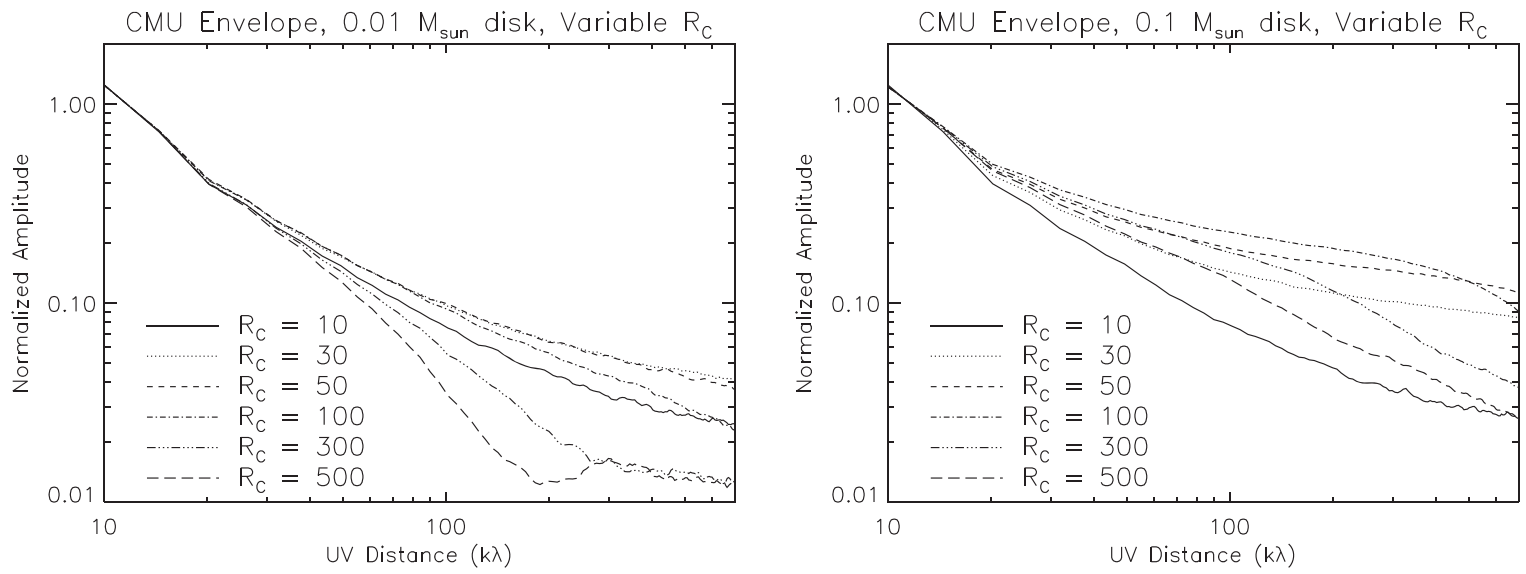

Figure 11. Visibility amplitudes for CMU Envelope models with disk components. The left panel shows CMU envelope models with an embedded disk of sizes between 10 and $500 \mathrm{AU}$ with a total mass of $0.01 M_{\odot}$. The right panel shows CMU envelope models with disks having a total mass of $0.1 M_{\odot}$. The envelopes have a total mass of $5.25 M_{\odot}$.

all have comparable resolution and sensitivity, a feature lacking from previous studies.

\subsection{Disk Formation}

The formation of disks during the protostellar phase has been thought to occur as a consequence of angular momentum conservation during protostellar collapse (Ulrich 1976; Cassen \& Moosman 1981; Terebey et al. 1984). This would enable the rapid formation of large disks during the protostellar phase, dependent entirely on the angular momentum inherited from the infalling envelope. Hydrodynamic simulations, without magnetic fields, readily form large disks during collapse (e.g., Yorke \& Bodenheimer 1999). These disks are massive enough 

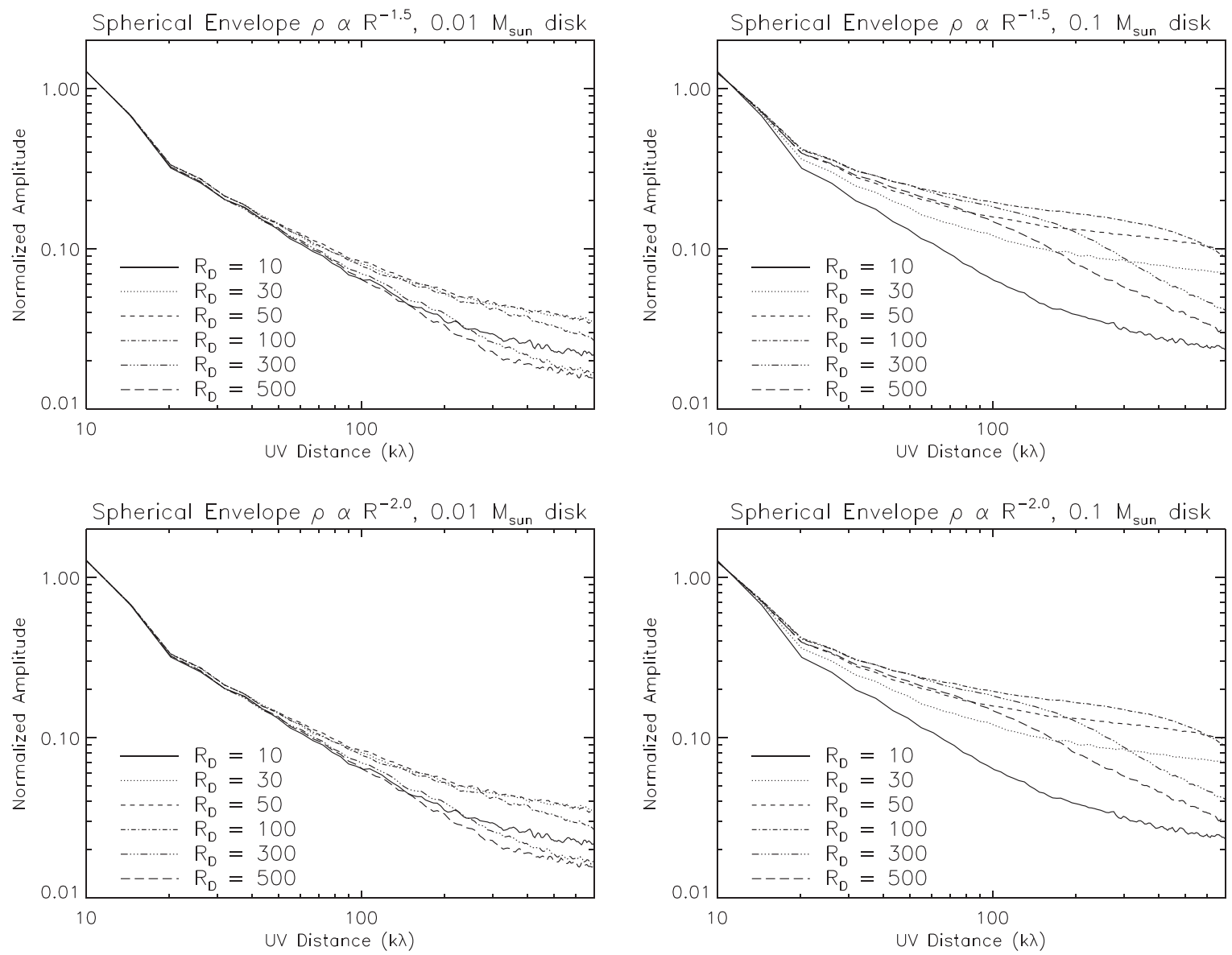

Figure 12. Visibility amplitudes for power-law Envelope models with disk components. The upper left panel shows $\rho \propto R^{-1.5}$ envelope models with embedded disks of sizes between 10 and $500 \mathrm{AU}$ with a total disk mass of $0.01 M_{\odot}$. The upper right panel is the same as the upper left, except that the disks have a total mass of 0.1 $M_{\odot}$. The lower left panel shows $\rho \propto R^{-2.0}$ envelope models with embedded disks having a total mass of $0.01 M_{\odot}$. The lower right panel is the same as the lower left, except that the disks have a total mass of $0.1 M_{\odot}$. The envelopes have a total mass of $5.25 M_{\odot}$.

to be gravitationally unstable, forming spiral arms and fragments (e.g., Boley 2009; Kratter et al. 2010; Vorobyov 2010; Zhu et al. 2012).

However, magnetic fields, which had been shown to potentially slow rotation during the pre-collapse phase (e.g., Basu 1998), were shown by Allen et al. (2003) to also significantly slow rotation during collapse and suppress the formation of a rotationally supported disk (Galli et al. 2006). These studies were verified by Mellon \& Li (2008) and Hennebelle \& Fromang (2008); disk formation was prevented even in the presence of very weak magnetic fields. More recent studies showed that by including non-ideal MHD effects in simulations, initially small rotationally supported disks could form (Dapp \& Basu 2010). The infalling material would still undergo magnetic braking, but the magnetic fields would be dissipated in the high-density material close to the protostar, enabling disk formation of a small rotationally supported disk. The size of the rotationally supported region will grow with time, but is expected to remain $<10 \mathrm{AU}$ throughout the Class 0 phase (Dapp et al. 2012). Machida et al. (2008) also simulated the formation of disks with non-ideal MHD and found that massive $100 \mathrm{AU}$ disks could form while in the Class 0 phase. A study by Joos et al. (2012) explored less ideal initial conditions, with misaligned magnetic fields and rotation axes. These simulations showed that even in the ideal MHD limit disks could form if the magnetic fields are misaligned with respect to the rotation axis.

The misaligned magnetic field scenario may be plausible because the TADPOL survey of magnetic field morphologies (Hull et al. 2013, 2014) found no systematic alignment of magnetic fields and outflow axes (presumed to reflect the rotation axis). Moreover, two of the four known Class 0 systems with rotationally supported disks (L1527 IRS and VLA 1623; Tobin et al. 2012; Murillo et al. 2013) were found to have magnetic fields perpendicular to the outflow axis on $\sim 1000$ AU scales and down to the scale of the disk in L1527 IRS (Segura-Cox et al. 2015). The sources L1448C, L1448 IRS3B, and L1448 IRS2 had observations in the TADPOL survey. L1448C only had a few detected magnetic field vectors, but they are at a $\sim 45^{\circ}$ angle to the outflow, L1448 IRS3B had vectors that are at an angle of $\sim 90^{\circ}$, and L1448 IRS2 had vectors that are both aligned and misaligned with respect to the outflow.

To summarize, theory predicts two different scenarios for disk formation, which depend on the included physics and the initial conditions, specifically the treatment and importance of magnetic fields. However, recent work with misaligned magnetic fields and non-ideal MHD (e.g., Tomida et al. 2015) seems to have reduced the significance of the "magnetic braking catastrophe." 

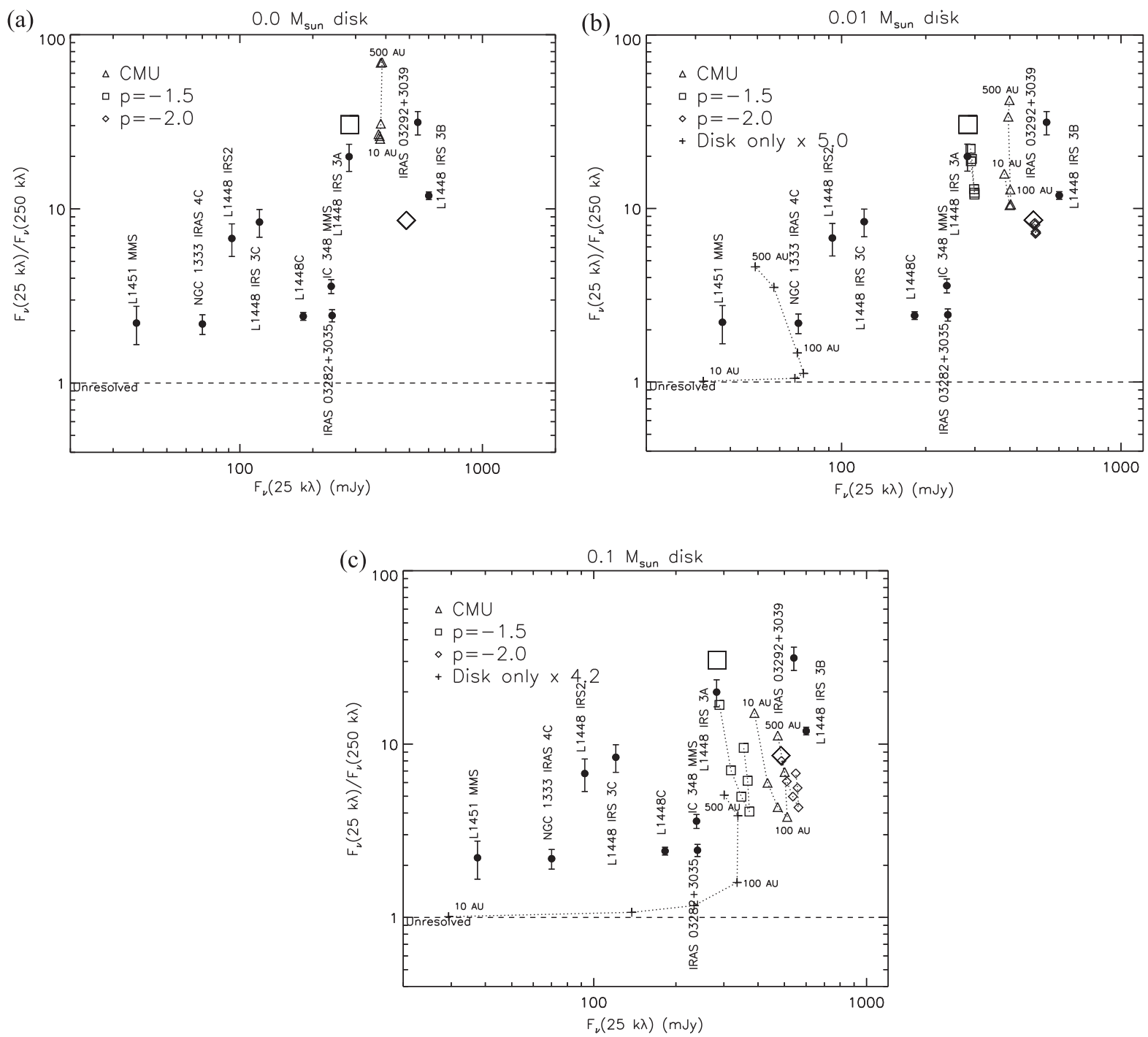

Figure 13. Visibility amplitude ratio plots for the visibility amplitudes at 25 and $250 \mathrm{k} \lambda$, corresponding to $10^{\prime \prime}(2300 \mathrm{AU})$ and $1^{\prime \prime}(230 \mathrm{AU})$ scales, respectively. The sources are plotted as the solid circles, and the models are plotted as the crosses (disk only), triangles (CMU models), squares $\left(\rho \propto R^{-1.5}\right)$, and diamonds $\left(\rho \propto R^{-2.0}\right)$. (a) Models without a disk component, but still a rotationally flattened region for the CMU case. (b) Models with a $0.01 M_{\odot}$ disk component (c) Models with a $0.1 M_{\odot}$ disk component. These plots imply that sources with a 25 -to- $250 \mathrm{k} \lambda$ ratio less than $\sim 8$ require a disk or another compact component, or a density profile steeper than $\rho$ $\propto R^{-2.0}$. Envelope models with an embedded disk can be made to have lower ratios by decreasing the envelope mass, emphasizing the disk component.The centrifugal/disk radii are labeled at 10,100, and $500 \mathrm{AU}$ for the CMU and disk models such that the variation of the visibility amplitude ratios can be traced. The $F_{\nu}$ $(25 \mathrm{k} \lambda)$ values for the disk-only models have been scaled by the factors noted in each plot such that the points for all radii are visible.

\subsection{Evidence for Class 0 Disks}

Thus far, rotationally supported disks have been detected toward four Class 0 protostars: L1527, VLA 1623, RCrA IRS7B, and HH212 MMS (Tobin et al. 2012; Murillo et al. 2013; Codella et al. 2014; Lindberg et al. 2014). L1527, VLA 1623, and RCrA IRS7B all have both extended dust emission perpendicular to the outflow direction and Keplerian rotation signature, while HH212 MMS is only resolved kinematically. It is unclear if the small number of observed Class 0 disks reflects a true paucity due to the physics of disk formation or if it is simply a lack of observations with high enough resolution and sensitivity to detect Class 0 protostellar disks (or disk candidates). Moreover, given that the emission from the disks is entangled with that of the envelope, they are more difficult to directly resolve than disks around Class I and Class II sources with much less (or no) envelope emission.
To increase the likelihood of detecting disks embedded within infalling envelopes, our sample is slightly biased toward sources that might have orientations that are within $30^{\circ}$ of edge-on and well-defined outflow axes from either infrared scattered light or $\mathrm{CO}$ emission. This is because edge-on disks should stand out better against the surrounding envelope due to the higher column density through the disk midplane. While our data are very sensitive and have a resolution of $\sim 0.3$ ( $70 \mathrm{AU})$, this is barely at the limit where we would expect to detect evidence for disks around Class 0 protostars. Indeed, for a source two beams across, the diameter would have to be at least $\sim 140 \mathrm{AU}$. The spatial resolution achieved by our survey is a factor of $\sim 1.6$ more coarse than the observations toward L1527 IRS in Tobin et al. (2012). If L1527 IRS was at the distance to Perseus, it would only be marginally resolved in our observations. 


\subsubsection{Sources with Evidence for $R>100$ AU Disks}

Despite the difficulties posed by limited spatial resolution, we do detect two strong candidate disks toward L1448 IRS2 and Per-emb-14 (NGC 1333 IRAS4C). The dust continuum structure around L1448 IRS2 is very large, 300 AU in radius. Per-emb-14 is more compact and has an apparent radius of $\sim 100$ AU.

The new and archival kinematic data for L1448 IRS2 did not enable a clear detection of Keplerian rotation around the protostar. Near the protostar, the velocity gradient in $\mathrm{C}^{18} \mathrm{O}$ only differs from the outflow position angle by $30^{\circ}$. The dust continuum emission at higher resolution is extended toward the southwest, and J. J. Tobin et al. (2015 in preparation) detect a binary source at the end of this dust extension. Takakuwa et al. (2014) showed that infall from a circumbinary disk, shepherded by the binary sources, could produce a radial velocity gradient along the outflow direction. Thus, the rotation signature on $>200$ AU scales could be that of a circumbinary disk, and the shift in velocity gradient direction could reflect material flowing through the disk toward the binary sources. On the other hand, a velocity gradient along the outflow direction is suspicious given that the outflow can entrain the ambient envelope material (Arce \& Sargent 2006). Thus, we merely suggest radial transport of material through the disk as a possibility to produce the velocity gradient along the outflow.

Per-emb-14 also appears to be a promising disk candidate from its resolved dust continuum structure. Our $\mathrm{C}^{18} \mathrm{O}$ data detected evidence of a velocity gradient in the expected direction for rotation. However, higher sensitivity will be required to verify this velocity gradient and determine if this rotation is Keplerian.

In addition to these two most promising $R>100 \mathrm{AU}$ disk candidates, IRAS $03292+3039$ has one of the brightest, largest, and most puzzling structures. While the position angle of this source is not close to being orthogonal to the outflow, the combined imaging from $\mathrm{C}$ and $\mathrm{B}$ configurations shows a 2'.5 (575 AU) diameter structure. Then at the highest resolution, the emission is rather constant across the source with a brightness temperature of $\sim 8 \mathrm{~K}$, indicating that the dust may be close to being optically thick, in which case the brightness temperature would reflect the temperature of the emitting material. In addition to the size of this structure, strong evidence of rotation on $\sim 3^{\prime \prime}$ scales was shown by Schnee et al. (2012) in $\mathrm{C}^{18} \mathrm{O}(J=2 \rightarrow 1)$ observations. Furthermore, the velocities of the outflow emission traced by $\mathrm{CO}(J=2 \rightarrow 1)$ indicate that this source is viewed at an intermediate inclination, possibly $40^{\circ}$ (Yen et al. 2015). The $\mathrm{C}^{18} \mathrm{O}$ $(J=2 \rightarrow 1)$ data were further analyzed by Yen et al. (2015), finding that a disk as large as $850 \mathrm{AU}$ could form in this system.

The large resolved structure, intermediate inclination, and rotation on scales comparable to the continuum size are evidence that this source may also be a good disk candidate, even though the continuum emission is not extended orthogonal to the outflow direction. The mass of the continuum source is also large, $\sim 0.2 M_{\odot}$ at the highest resolution, and the protostar mass is estimated to be $\sim 0.3 M_{\odot}$ (Yen et al. 2015). If the continuum source is indeed rotationally supported, the large ratio of disk to protostar mass could mean that the disk would be gravitationally unstable. However, future observations with higher resolution will be needed to verify if the continuum structure is a rotationally supported disk, as well as a more robust kinematic measurement of the protostar mass.

\subsubsection{Sources with Evidence for $R<100$ AU Disks}

While there is evidence for large disk-like structures around a few protostars, the results are not so clear for the remaining sources. The sources L1448C, IRAS 03282+3035, and L1448 IRS3C have deconvolved position angles that are $7^{\circ}, 19^{\circ}$, and $9^{\circ}$ from being orthogonal to the outflow, respectively. However, the continuum emission is not obviously disk-like in the images, but only higher-resolution/sensitivity data will be able to determine this for certain, in addition to molecular line observations.

The analysis of visibility amplitude ratios enables us to shed further light on the evidence for disks (or at least compact dust structures) on scales smaller than 100 AU. Figure 13 shows that sources with 25 -to- $250 \mathrm{k} \lambda$ amplitude ratios less than $\sim 8$ require a contribution from a compact component, possibly a disk. A ratio of 8 is the value for a spherical envelope with a radial density profile $\rho \propto R^{-2}$ and no compact density structure, the steepest we might expect in idealized star formation models; the ratio of an $R^{-1.5}$ density profile is $\sim 30$. The ratio expected for a 500 AU disk itself is $\sim 5$. Thus, the ratio between 0 and 8 will depend on both the radius of the disk and the amount of mass in the disk relative to the envelope.

L1451-MMS, Per-emb-14, L1448C, IC 348 MMS, L1448 IRS2, and IRAS $03282+3035$ all have a 25 -to- $250 \mathrm{k} \lambda$ ratio less than 8 (six out of nine Class 0 objects in the sample). L1448 IRS3C was discussed previously as having resolved emission nearly orthogonal to the outflow, but it does not have a ratio less than 8 . This could result from its close proximity to L1448 IRS3B, given that its visibility amplitudes have unexplained dips at 200 and $40 \mathrm{k} \lambda$. IRAS $03292+3039$ (discussed in the previous section) also has a ratio $>8$, but this could be caused by the emission from the disk and inner envelope being optically thick out to $>1^{\prime \prime}$ scales, leading to the emission being resolved out.

L1448 IRS3B appears to have extended envelope emission orthogonal to the outflow, encompassing the two sources. The detected emission is not significantly larger in angular extent than that of L1448 IRS2 and IRAS $03292+3039$, but the emission is not centered on either of the continuum sources. Kinematic observations of L1448 IRS3B by Yen et al. (2015) show that there is a velocity gradient consistent with rotation on $3^{\prime \prime}$ scales, but higher-resolution kinematic observations will be needed to determine the nature of this structure.

\subsubsection{Outlook}

The results from this small survey have shown that at least two (Per-emb-14 and L1448 IRS2), possibly three (including IRAS 03292+3039), show evidence for resolved disks with radii $>100 \mathrm{AU}$. The remaining sources have evidence for compact structure within their envelopes that could be evidence for a disk, but higher-resolution continuum and kinematic observations are necessary to characterize them. Despite the small sample, it is encouraging for studies of Class 0 disks that three of nine Class 0 sources have evidence for resolved disklike structure and many of the others have compact emission extended perpendicular to their outflows or visibility amplitude ratios that suggest a disk-like component. While we cannot 
prove that the disk candidates are rotationally supported, it is important to highlight that we are detecting an abundance of structure on scales less than $500 \mathrm{AU}$ toward Class 0 protostars, possibly a signature of protostellar disks.

\subsection{Class O Disk Masses}

Assuming that we are probing emission from the protostellar disks, we have been able to calculate their masses to compare with measurements of other Class 0 sources and more evolved Class I and II objects. The median masses of the compact components are $\sim 0.05 M_{\odot}$ (values corrected for estimated envelope emission) for measurements taken at both 50 and $100 \mathrm{k} \lambda$. This mass is an order of magnitude larger than the characteristic mass of Class II disks $\left(\sim 0.005 M_{\odot}\right.$; Andrews \& Williams 2005) and $5 \times$ larger than the median Class I disk mass measured in Jørgensen et al. (2009). The Class 0 disk masses measured in Jørgensen et al. (2009) did have some sources with even larger masses than we find, which may support a scenario of typically higher disk masses in the Class 0 phase. A caveat of this comparison is that while Jørgensen et al. (2009) assumed the same dust opacities as our study, Andrews $\&$ Williams (2005) assumed $\kappa_{850 \mu \mathrm{m}}=0.035$ (dust plus gas opacity), about a factor of two larger than the Ossenkopf \& Henning (1994) model at $850 \mu \mathrm{m}$ (a factor of two larger opacity decreases the calculated mass by the same factor). However, a larger opacity might be more appropriate for Class II disks, but more importantly the gas-to-dust mass ratio in Class II disks might be significantly less than 100 (Williams \& Best 2014). The gas-to-dust ratio uncertainty is potentially more significant than the opacity uncertainty in the case of Class II disks, while the gas and dust may still be well mixed in the Class 0 phase.

The main uncertainty in masses derived in this analysis is the true envelope density structure. Numerous studies have shown that Class 0 envelopes may have a variety of density profiles; the most typical profiles are steeper than $\rho \propto R^{-1.5}$ (Looney et al. 2003; Chiang et al. 2008; Kwon et al. 2009; Chiang et al. 2012; Tobin et al. 2015). This is important, because steeper envelope density profiles result in greater contributions of envelope emission at 50 and $100 \mathrm{k} \lambda$, leading to overestimated disk masses.

\subsection{Multiplicity}

Our sample is not large enough to make strong revisions to the current multiplicity result toward Class 0 protostars, which was recently found to be 0.64 by Chen et al. (2013). We have discovered one new close companion (L1448 IRS3B separated by $210 \mathrm{AU}$ ), and we believe that the $300 \mathrm{AU}$ companion toward IRAS $03282+3035$ is spurious. Thus, the multiplicity fraction as a whole does not change. However, we did sensitively sample smaller spatial scales than previous studies and only confidently find one new clear candidate companion (L1448 IRS3B). IRAS 03292+3035 may be another candidate, but its multiplicity is uncertain.

Maury et al. (2010) had suggested a lack of multiplicity on scales between 150 and $430 \mathrm{AU}$ (corrected for the updated distance to Perseus), but our new binary detection is separated by $210 \mathrm{AU}$, and the sample of Chen et al. (2013) was quite incomplete at those scales. A larger, less-biased sample will better illuminate the multiplicity frequency as a function of separation; such a project is being carried out now with the VLA (J. J. Tobin et al. 2015, in preparation).

\section{SUMMARY}

We have presented a CARMA $1.3 \mathrm{~mm}$ survey of nine Class 0 protostars and two Class I protostars in the Perseus molecular cloud. This is one of the largest high-resolution $(\sim 0.3,70 \mathrm{AU})$ samples of $1.3 \mathrm{~mm}$ data taken toward Class 0 protostars (thus far). We also include kinematic follow-up for two sources (Peremb-14 and L1448 IRS2). The main results from the survey can be summarized as follows.

(1) We detect three strong Class 0 disk candidates toward L1448 IRS2, Per-emb-14, and IRAS 03292+3039. These systems are not yet kinematically confirmed to be Keplerian disks. L1448 IRS2 shows evidence of rotation on scales $>200 \mathrm{AU}$, with a change in velocity gradient direction on scales <200 AU. Per-emb-14 has an indication of rotation on the scale of the detected disklike structure, but the spectral line data have low $\mathrm{S} / \mathrm{N}$, and we cannot determine if the rotation is Keplerian. IRAS $03292+3039$ does show evidence for rotation on 500 AU scales, but smaller-scale measurements are not yet available.

(2) A variety of resolved structures are detected within our continuum data, aside from the disk candidates listed in point 1, ranging from apparent massive inner envelopes or disks (L1448 IRS3B and IRAS 03292+3039) to marginally resolved structures on $<200 \mathrm{AU}$ scales (IRAS 03282+3035, L1448 IRS3C). Finally, three sources do not appear to have resolved structure on scales <200 AU (L1448C, IC 348 MMS, L1451-MMS). Moreover, five out of nine sources in the sample have deconvolved position angles that are within $30^{\circ}$ of orthogonal to the outflow, possibly an indication of compact structure consistent with a disk.

(3) Comparison of the observed visibility amplitude ratios to radiative transfer models of disks and envelopes enables us to infer that at least six of nine sources in our sample require a compact component, possibly represented by a disk (L1451-MMS, Per-emb-14, L1448C, IC 348 MMS, L1448 IRS2, and IRAS 03282+3035). The radiative transfer models also show that emission from embedded disks with masses $\sim 0.1 M_{\odot}$ and radii $<30 \mathrm{AU}$ will be optically thick and have lower levels of emission at $1.3 \mathrm{~mm}$ relative to larger disks with the same mass.

(4) A candidate companion to L1448 IRS3B is detected with a separation of 0.9 ( $\sim 210 \mathrm{AU})$, and the companion itself may be composed of two sources separated by $\sim 0.2$. IRAS 03292+3039 may have multiple components, but the multiple peaks detected do not significantly stand out from the resolved structure. We do not detect a companion source toward IRAS $03282+3035$ that had been detected by Chen et al. (2013).

We conclude that there is evidence for the formation of large disks in the Class 0 phase, but Class 0 disks likely have a range of radii and masses that depend on the structure, kinematics, and possibly magnetic field properties of their parent cores. Sub-arcsecond-resolution imaging is crucial to characterizing the structure of Class 0 disks, and observations with ALMA and the VLA at wavelengths longer than $1.3 \mathrm{~mm}$ will likely be necessary to characterize emission from small, massive disks. 

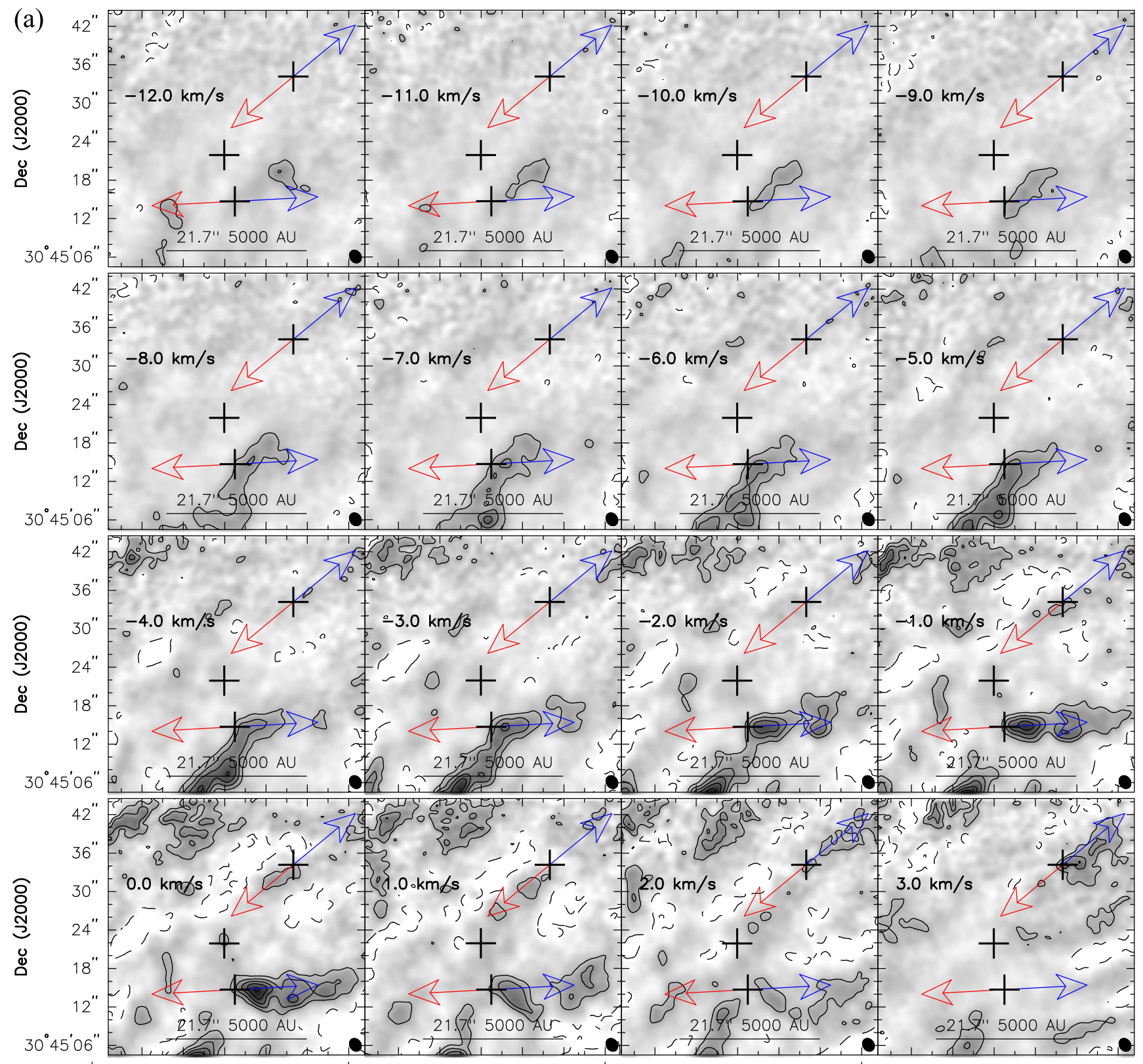

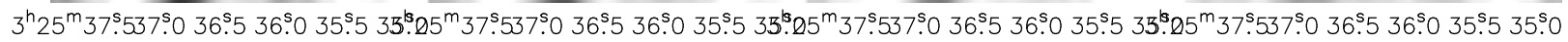

RA (J2000)

RA (J2000)

RA (J2000)

RA (J2000)

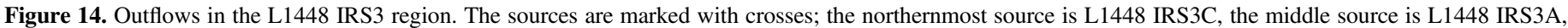

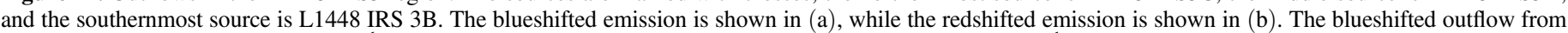

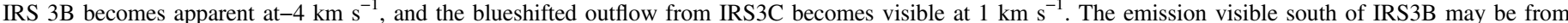

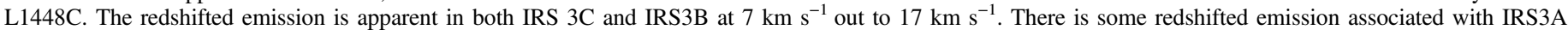

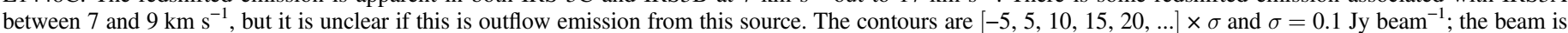
$2.1 \times 1.7$. The blue and red arrows denote the direction of the blueshifted and redshifted outflows, respectively.

We wish to thank the anonymous referee for helpful comments that have improved the clarity of this paper. We also wish to thank Mike Dunham and Jes Jorgensen for useful discussions. J.J.T. acknowledges support provided by NASA through Hubble Fellowship grant \#HST-HF-51300.01 A awarded by the Space Telescope Science Institute, which is operated by the Association of Universities for Research in Astronomy, Inc., for NASA, under contract NAS 5-26555. This work is supported by grant 639.041.439 from the Netherlands Organisation for Scientific Research (NWO). This work is supported by the European Union A-ERC grant 291141 CHEMPLAN. L.L. acknowledges the support of
DGAPA, UNAM, CONACyT (México), for financial support. Support for CARMA construction was derived from the states of Illinois, California, and Maryland, the James S. McDonnell Foundation, the Gordon and Betty Moore Foundation, the Kenneth T. and Eileen L. Norris Foundation, the University of Chicago, the Associates of the California Institute of Technology, and the National Science Foundation. Ongoing CARMA development and operations are supported by the National Science Foundation under a cooperative agreement, and by the CARMA partner universities. The SMA is a joint project between the Smithsonian Astrophysical Observatory and the Academia Sinica Institute of Astronomy and Astrophysics and is 


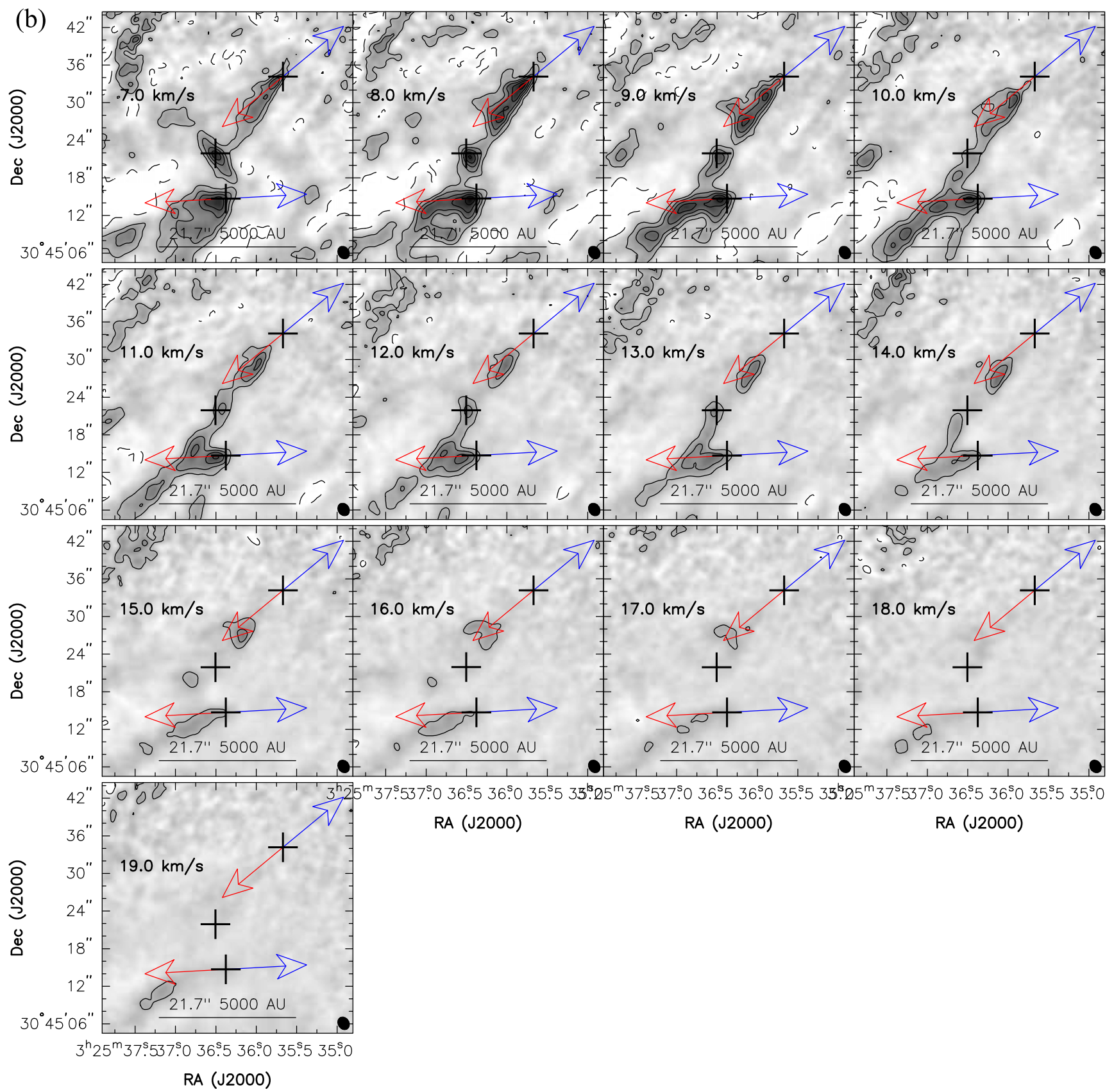

Figure 14. (Continued.)

funded by the Smithsonian Institution and the Academia Sinica. The National Radio Astronomy Observatory is a facility of the National Science Foundation operated under cooperative agreement by Associated Universities, Inc. This research made use of APLpy, an open-source plotting package for Python hosted at http://aplpy.github.com. This research has made use of NASA's Astrophysics Data System Bibliographic Services.

The authors wish to recognize and acknowledge the very significant cultural role and reverence that the summit of Mauna Kea has always had within the indigenous Hawaiian community. We are most fortunate to have the opportunity to conduct observations from this mountain.

Facilities: CARMA, SMA, Spitzer.

\section{APPENDIX \\ OUTFLOW MAPS}

Our observations included the ${ }^{12} \mathrm{CO}$ and ${ }^{13} \mathrm{CO}(J=2 \rightarrow 1)$ transitions that, when detected, enabled us to examine the outflow structure toward some of our protostellar targets at resolutions that are higher than typically achieved for outflow emission.

\section{A.1 L1448 IRS3}

The L1448 IRS3 region is of particular interest because of the complex, overlapping outflows in the region (Kwon et al. 2006), and there have not been higher angular resolution observations toward these sources. We show the outflow channel maps in Figure 14 from a combination of our higher- 

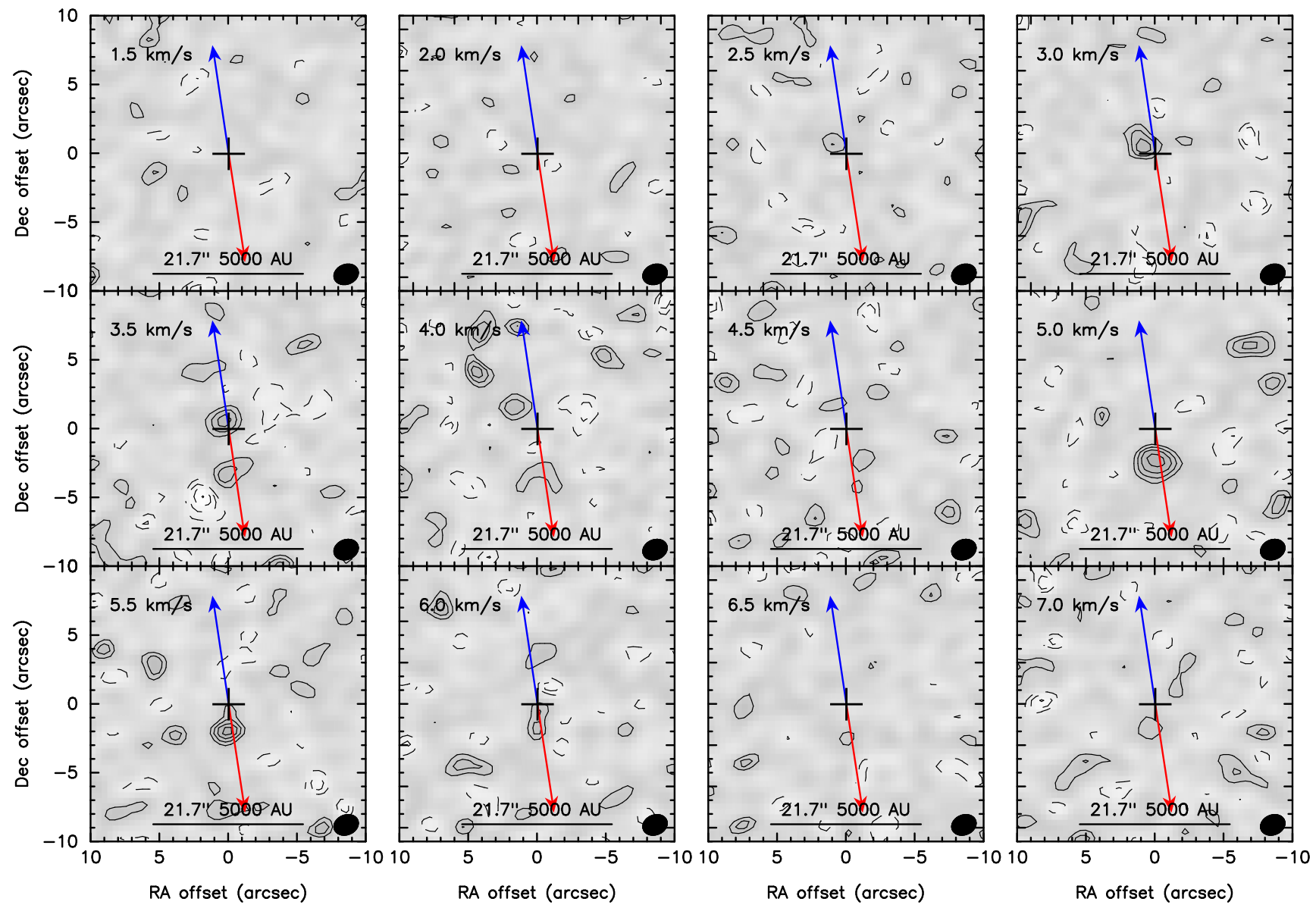

Figure 15. Compact outflow from L1451-MMS. The outflow is detected to be in the same direction as observed by Pineda et al. (2011) and with a similar offset to the east of the $1.3 \mathrm{~mm}$ continuum peak. The contours are $\pm[2,3,4,5] \times \sigma$ and $\sigma=0.1 \mathrm{Jy}^{\mathrm{m}} \mathrm{beam}^{-1}$; the beam is $0.92 \times 0.72$. The blue and red arrows denote the direction of the blueshifted and redshifted outflows, respectively.

resolution data and the CO data observed in CARMA D-array by Hull et al. (2014), gaining better sensitivity to extended structures. The sources are marked with crosses, and we can clearly identify the outflow of L1448 IRS3B, the southernmost source. There appears to be a contribution from the L1448C outflow in the southern parts of the map. We also can identify, for the first time, an outflow associated with the northernmost source, L1448 IRS3C. For this source, we mainly detect the redshifted side of the outflow, and the blueshifted side is observed in only a few velocity channels. Nevertheless, both the blue- and redshifted sides of the outflow have a cone-like morphology that can be traced back to an origin at the position of L1448 IRS3C. The asymmetric brightness of the blue- and redshifted outflow components may result from L1448 IRS3C being located near the edge of the L1448 cloud and having less entrained material on the blueshifted side.

Kwon et al. (2006) had associated what we now believe to be the redshifted side of the L1448 IRS3C outflow with L1448 IRS3A, the more evolved Class I protostar in the region. However, the bipolar pattern of the $\mathrm{CO}$ emission seems to clearly originate at the position of IRS3C. We do see some redshifted CO emission near L1448 IRS3A that seems to be extended toward the northeast; however, we cannot clearly identify a position angle for this outflow. Scattered light morphology shown by Tobin et al. (2007) may suggest that the outflow has a slight northwest position angle, but the complexities of the emission from the three protostars within $30^{\prime \prime}$ of each other make such an identification difficult.

\section{A.2 L1451-MMS}

We detected CO $(J=2 \rightarrow 1)$ toward L1451-MMS, the candidate first hydrostatic core identified by Pineda et al. (2011), who also found a compact CO outflow in their lowerresolution data. We confirm the presence of the $\mathrm{CO}$ outflow with our higher-resolution data as shown in the channel maps in Figure 15. Moreover, we also find that the continuum emission is slightly offset from the line connecting the blueand redshifted outflow; this slight offset was also seen in the data from Pineda et al. (2011). The offset may be related to the outflow being driven into an inhomogeneous medium. This offset is also in the direction of the $3 \sigma$ extension seen in the continuum emission. The detection of the outflow with our high-resolution data demonstrates the compactness of the outflow; otherwise, it would have been resolved out.

\section{A.3 L1448 IRS2}

We detect the outflow toward L1448 IRS2 in CO $(J=2 \rightarrow 1)$, as shown in Figure 16, with excellent sensitivity. The high resolution and sensitivity are achieved with a combination of SMA Compact and Extended configurations, as well as CARMA C and B configurations. The outflow is clearly offset from the $1.3 \mathrm{~mm}$ continuum peak by about $1^{\prime \prime}$, 


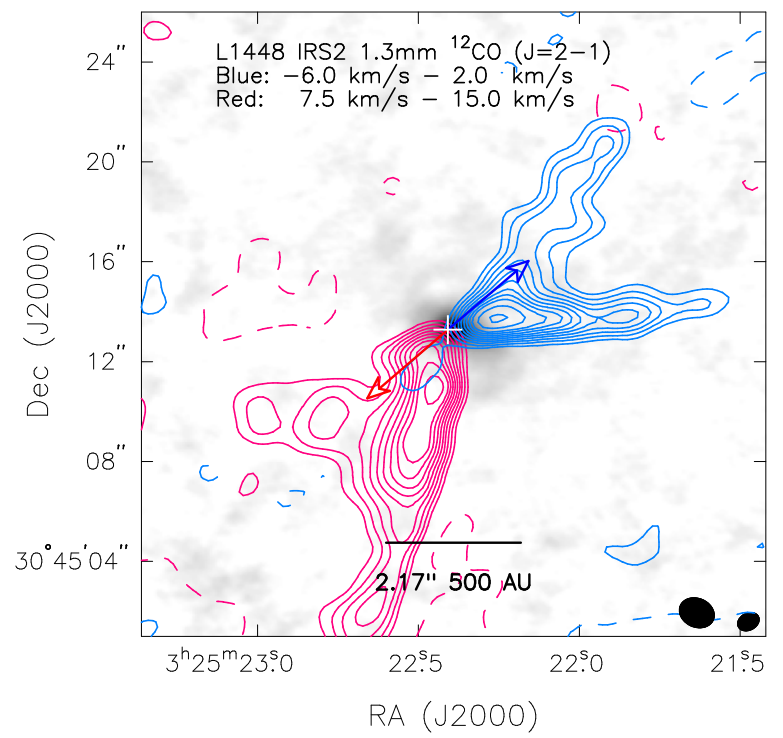

Figure 16. Integrated intensity maps of ${ }^{12} \mathrm{CO}$ emission toward L1448 IRS2 from the combined imaging of CARMA and SMA data sets, overlaid on the CARMA $1.3 \mathrm{~mm}$ continuum map (grayscale). The emission maps reveal a rather asymmetric appearance of the outflow cavities, opening wider to the southwest in comparison to the northeast. Furthermore, the outflow vectors, which are centered on the peak of the continuum emission, seem as if they would be better centered slightly to the southwest, along the extended continuum emission. The contours are $[-6,6,9,12,15,18, \ldots] \times \sigma$, and $\sigma=2.47$ and $2.55 \mathrm{~K}$ for the red- and blueshifted integrated intensity maps, respectively. The beam for the CO emission is $1.42 \times 1.13$, and the beam for the continuum is $0.91 \times 0.68$. The blue and red arrows denote the direction of the blueshifted and redshifted outflows, respectively.

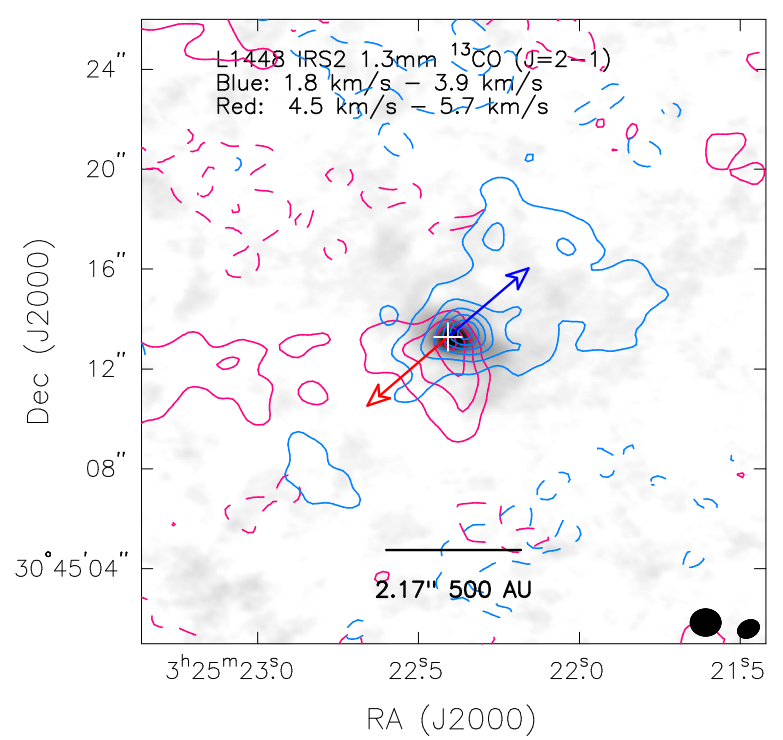

Figure 17. Integrated intensity maps of ${ }^{13} \mathrm{CO}$ emission toward L1448 IRS2 from the combined imaging of CARMA and SMA data sets, overlaid on the CARMA $1.3 \mathrm{~mm}$ continuum map (grayscale). In the case of L1448 IRS2, the ${ }^{13} \mathrm{CO}$ emission is tracing the dense, lower-velocity outflow. The positional offset of the blueshifted emission is consistent with that of the $\mathrm{C}^{18} \mathrm{O}$ emission shown in Figure 5. The contours are $[-3,3,6,9,12,15,18, \ldots] \times \sigma$, and $\sigma=1.14$ and $1.44 \mathrm{~K}$ for the red- and blueshifted integrated intensity maps, respectively. The beam for the $\mathrm{CO}$ emission is $1.2 \times 1.06$, and the beam for the continuum is $0.91 \times 0.68$. The blue and red arrows denote the direction of the blueshifted and redshifted outflows, respectively.

and the outflow cavities appear asymmetric, opening wider to the southwest than the northeast. This asymmetry is evident in both the blue- and redshifted lobes of the outflow. The asymmetry in the opening angle could reflect the structure of

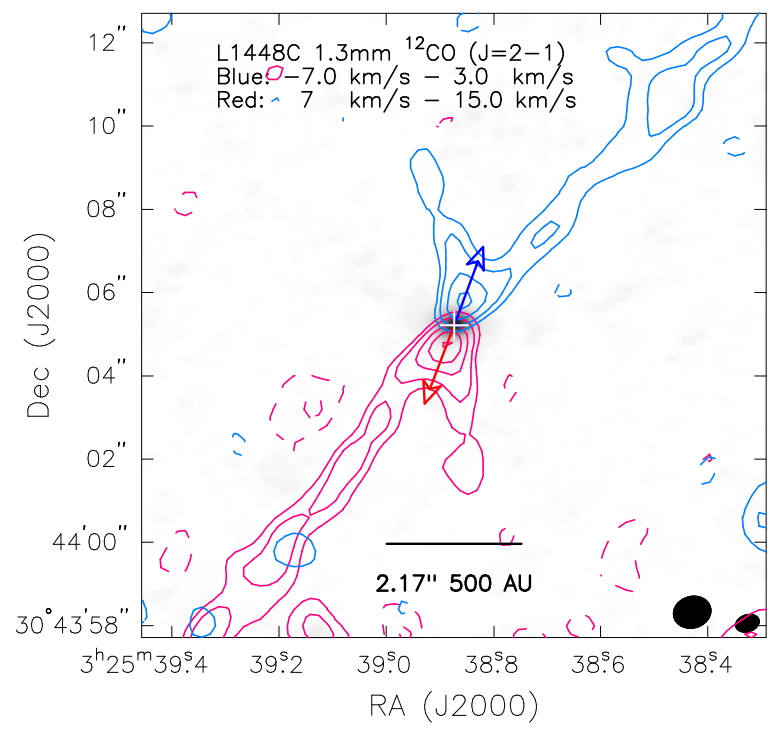

Figure 18. Integrated intensity maps of ${ }^{12} \mathrm{CO}$ emission toward L1448C from the CARMA B- and C-array data, overlaid on the CARMA $1.3 \mathrm{~mm}$ continuum map (grayscale). The contours are $[-4,46,9,12,15,18, \ldots] \times \sigma$, and $\sigma=13.922$ and $15.39 \mathrm{~K}$ for the red- and blueshifted integrated intensity maps, respectively. The beam for the $\mathrm{CO}$ emission is $0.90 \times 0.75$, and the beam for the continuum is $0.58 \times 0.41$. The blue and red arrows denote the direction of the blueshifted and redshifted outflows, respectively.

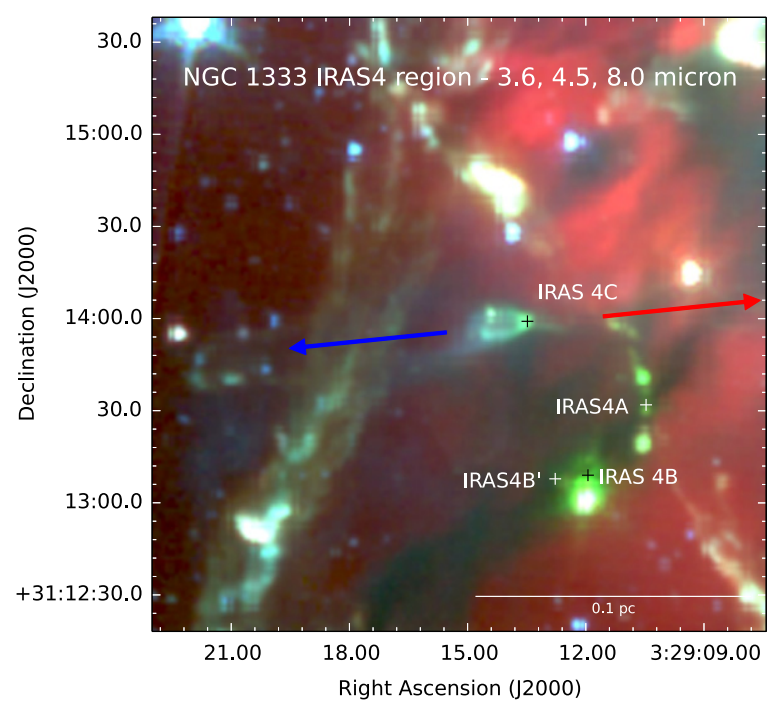

Figure 19. Spitzer IRAC $3.6 \mu \mathrm{m}, 4.5 \mu \mathrm{m}$, and $8.0 \mu \mathrm{m}$ imaging of the NGC 1333 IRAS4 region. The blue and red vectors mark the outflow position angle adopted for Per-emb-14 (NGC 1333 IRAS4C).

the ambient medium in the direct vicinity of the protostars, with the envelope being less dense to the southwest and enabling the cavities to open more widely. Furthermore, the outflow would appear more symmetric if the driving source was shifted southwest a few arcseconds, along the extended dust continuum emission.

The combined sensitivity of the CARMA and SMA maps also enabled ${ }^{13} \mathrm{CO}(J=2 \rightarrow 1)$ to be resolved in the outflow toward L1448 IRS2 (Figure 17). The ${ }^{13} \mathrm{CO}$ emission traces more compact structure located near the continuum source, but the asymmetry and spatial offset evident in the ${ }^{12} \mathrm{CO}$ emission are still present. Moreover, the location of the blueshifted intensity peak is coincident with the blueshifted intensity peak in $\mathrm{C}^{18} \mathrm{O}$, evidence that the compact $\mathrm{C}^{18} \mathrm{O}$ may be affected by the outflow. 


\section{A.4 L1448C}

Lastly, we were able to detect the compact inner outflow toward L1448C in CO $(J=2 \rightarrow 1)$ with only the CARMA B- and C-array data. We were able to obtain a good detection with such little time on source due to L1448C having extremely bright $\mathrm{CO}$ emission. Maps with superior sensitivity and comparable resolution do exist (e.g., Hirano et al. 2010), but we include the map in Figure 18 for the sake of completeness.

\section{A.5 Per-emb-14}

Per-emb-14 does not currently have a $\mathrm{CO}$ outflow detection, possibly due to the source being edge-on. However, the Spitzer IRAC images shown in Figure 19 of the source reveal a scattered light nebula at the position of the source, along with shock features extended along the inferred blueshifted side of the outflow. These data were taken as part of Spitzer GO program 30516 and were reduced with the same methods described in Tobin et al. (2010b) and are more sensitive than the data presented by Gutermuth et al. (2008). We infer that the eastern side of the outflow is blueshifted because it is brighter and it is known that the geometrical effects will cause the redshifted side to be more extincted and have a fainter nebula (Whitney et al. 2003).

\section{REFERENCES}

Allen, A., Li, Z., \& Shu, F. H. 2003, ApJ, 599, 363

Andre, P., Ward-Thompson, D., \& Barsony, M. 1993, ApJ, 406, 122

Andrews, S. M., \& Williams, J. P. 2005, ApJ, 631, 1134

Arce, H. G., \& Sargent, A. I. 2006, ApJ, 646, 1070

Basu, S. 1998, ApJ, 509, 229

Beckwith, S. V. W., Sargent, A. I., Chini, R. S., \& Guesten, R. 1990, AJ, 99, 924

Boley, A. C. 2009, ApJL, 695, L53

Bourke, T. L., Myers, P. C., Evans, N. J., II, et al. 2006, ApJL, 649, L37

Brown, D. W., Chandler, C. J., Carlstrom, J. E., et al. 2000, MNRAS, 319, 154

Cassen, P., \& Moosman, A. 1981, Icar, 48, 353

Chandler, C. J., Koerner, D. W., Sargent, A. I., \& Wood, D. O. S. 1995, ApJL, 449, L139

Chen, X., Arce, H. G., Zhang, Q., et al. 2010, ApJ, 715, 1344

Chen, X., Arce, H. G., Zhang, Q., et al. 2013, ApJ, 768, 110

Chiang, H., Looney, L. W., Tassis, K., Mundy, L. G., \& Mouschovias, T. C. 2008, ApJ, 680, 474

Chiang, H., Looney, L. W., \& Tobin, J. J. 2012, ApJ, 709, 470

Codella, C., Cabrit, S., Gueth, F., et al. 2014, A\&A, 568, L5

Curtis, E. I., Richer, J. S., Swift, J. J., \& Williams, J. P. 2010, MNRAS, 408, 1516

Dapp, W. B., \& Basu, S. 2010, A\&A, 521, L56+

Dapp, W. B., Basu, S., \& Kunz, M. W. 2012, A\&A, 541, A35

Dunham, M. M., Evans, N. J., II, Bourke, T. L., et al. 2006, ApJ, 651, 945

Enoch, M. L., Evans, N. J., Sargent, A. I., \& Glenn, J. 2009, ApJ, 692, 973

Enoch, M. L., Young, K. E., Glenn, J., et al. 2006, ApJ, 638, 293

Frank, A., Ray, T. P., Cabrit, S., et al. 2014, in Protostars and Planets VI, ed. H. Beuther, R. S. Klessen, C. P. Dullemond, \& T. Henning (Tucson, AZ: Univ. Arizona Press), 451

Galli, D., Lizano, S., Shu, F. H., \& Allen, A. 2006, ApJ, 647, 374

Gueth, F., Guilloteau, S., \& Bachiller, R. 1996, A\&A, 307, 891

Gutermuth, R. A., Myers, P. C., Megeath, S. T., et al. 2008, ApJ, 674, 336

Harvey, D. W. A., Wilner, D. J., Myers, P. C., \& Tafalla, M. 2003, ApJ, 596,383

Hennebelle, P., \& Fromang, S. 2008, A\&A, 477, 9

Hirano, N., Ho, P. P. T., Liu, S.-Y., et al. 2010, ApJ, 717, 58

Hirano, N., Kamazaki, T., Mikami, H., Ohashi, N., \& Umemoto, T. 1999, in Star Formation, ed. T. Nakamoto (Nagano: Nobeyama Radio Observatory), 181

Hirota, T., Bushimata, T., Choi, Y. K., et al. 2008, PASJ, 60, 37

Hirota, T., Honma, M., Imai, H., et al. 2011, PASJ, 63, 1

Hull, C. L. H., Plambeck, R. L., Bolatto, A. D., et al. 2013, ApJ, 768, 159

Hull, C. L. H., Plambeck, R. L., Kwon, W., et al. 2014, ApJS, 213, 13

Joos, M., Hennebelle, P., \& Ciardi, A. 2012, A\&A, 543, A128
Jørgensen, J. K., Harvey, P. M., Evans, N. J., II, et al. 2006, ApJ, 645, 1246

Jørgensen, J. K., van Dishoeck, E. F., Visser, R., et al. 2009, A\&A, 507, 861

Kratter, K. M., Matzner, C. D., Krumholz, M. R., \& Klein, R. I. 2010, ApJ, 708,1585

Kwon, W., Looney, L. W., Crutcher, R. M., \& Kirk, J. M. 2006, ApJ, 653,1358

Kwon, W., Looney, L. W., Mundy, L. G., Chiang, H.-F., \& Kemball, A. J. 2009, ApJ, 696, 841

Larson, R. B. 1969, MNRAS, 145, 271

Lazarian, A., Esquivel, A., \& Crutcher, R. 2012, ApJ, 757, 154

Li, Z.-Y., Krasnopolsky, R., \& Shang, H. 2013, ApJ, 774, 82

Lindberg, J. E., Jørgensen, J. K., Brinch, C., et al. 2014, A\&A, 566, A74

Looney, L. W., Mundy, L. G., \& Welch, W. J. 2000, ApJ, 529, 477

Looney, L. W., Mundy, L. G., \& Welch, W. J. 2003, ApJ, 592, 255

Machida, M. N., Tomisaka, K., Matsumoto, T., \& Inutsuka, S. 2008, ApJ, 677, 327

Maury, A. J., André, P., Hennebelle, P., et al. 2010, A\&A, 512, A40

McMullin, J. P., Waters, B., Schiebel, D., Young, W., \& Golap, K. 2007, in ASP Conf. Ser. 376, Astronomical Data Analysis Software and Systems XVI, ed. R. A. Shaw, F. Hill, \& D. J. Bell (San Francisco, CA: ASP), 127 Mellon, R. R., \& Li, Z.-Y. 2008, ApJ, 681, 1356

Murillo, N. M., Lai, S.-P., Bruderer, S., Harsono, D., \& van Dishoeck, E. F. 2013, A\&A, 560, A103

Ohashi, N., Saigo, K., Aso, Y., et al. 2014, ApJ, 796, 131

Ormel, C. W., Min, M., Tielens, A. G. G. M., Dominik, C., \& Paszun, D. 2011, A\&A, 532, A43

Ossenkopf, V., \& Henning, T. 1994, A\&A, 291, 943

Padovani, M., Hennebelle, P., \& Galli, D. 2013, A\&A, 560, A114

Palau, A., Zapata, L. A., Rodríguez, L. F., et al. 2014, MNRAS, 444, 833

Pech, G., Zapata, L. A., Loinard, L., \& Rodríguez, L. F. 2012, ApJ, 751, 78

Pérez, L. M., Carpenter, J. M., Chandler, C. J., et al. 2012, ApJL, 760, L17

Pérez, L. M., Lamb, J. W., Woody, D. P., et al. 2010, ApJ, 724, 493

Pineda, J. E., Arce, H. G., Schnee, S., et al. 2011, ApJ, 743, 201

Plunkett, A. L., Arce, H. G., Corder, S. A., et al. 2013, ApJ, 774, 22

Raghavan, D., McAlister, H. A., Henry, T. J., et al. 2010, ApJS, 190, 1

Reid, M. J., Schneps, M. H., Moran, J. M., et al. 1988, ApJ, 330, 809

Robitaille, T. P. 2011, A\&A, 536, A79

Rodríguez, L. F., Zapata, L. A., \& Palau, A. 2014, ApJ, 790, 80

Sadavoy, S. I., Di Francesco, J., André, P., et al. 2014, ApJL, 787, L18

Sakai, N., Oya, Y., Sakai, T., et al. 2014a, ApJL, 791, L38

Sakai, N., Sakai, T., Hirota, T., et al. 2014b, Natur, 507, 78

Sault, R. J., Teuben, P. J., \& Wright, M. C. H. 1995, in ASP Conf. Ser. 77, Astronomical Data Analysis Software and Systems IV, ed. R. A. Shaw, H. E. Payne, \& J. J. E. Hayes (San Francisco, CA: ASP), 433

Schnee, S., Sadavoy, S., Di Francesco, J., Johnstone, D., \& Wei, L. 2012, ApJ, 755,178

Segura-Cox, D. M., Looney, L. W., Stephens, I. W., et al. 2015, ApJL, 798, L2

Seifried, D., Banerjee, R., Pudritz, R. E., \& Klessen, R. S. 2013, MNRAS, 432,3320

Shakura, N. I., \& Syunyaev, R. A. 1973, A\&A, 24, 337

Shirley, Y. L., Mason, B. S., Mangum, J. G., et al. 2011, AJ, 141, 39

Shu, F. H. 1977, ApJ, 214, 488

Smith, K. W., Bonnell, I. A., Emerson, J. P., \& Jenness, T. 2000, MNRAS, 319,991

Takakuwa, S., Saito, M., Saigo, K., et al. 2014, ApJ, 796, 1

Terebey, S., \& Padgett, D. L. 1997, in IAU Symp. 182, Herbig-Haro Flows and the Birth of Stars, ed. B. Reipurth, \& C. Bertout (Dordrecht: Kluwer), 507

Terebey, S., Shu, F. H., \& Cassen, P. 1984, ApJ, 286, 529

Tobin, J. J., Hartmann, L., Chiang, H.-F., et al. 2012, Natur, 492, 83

Tobin, J. J., Hartmann, L., Chiang, H.-F., et al. 2013, ApJ, 771, 48

Tobin, J. J., Hartmann, L., \& Loinard, L. 2010a, ApJL, 722, L12

Tobin, J. J., Hartmann, L., Looney, L. W., \& Chiang, H. 2010b, ApJ, 712,1010

Tobin, J. J., Looney, L. W., Mundy, L. G., Kwon, W., \& Hamidouche, M. 2007, ApJ, 659, 1404

Tobin, J. J., Stutz, A. M., Megeath, S. T., et al. 2015, ApJ, 798, 128

Tomida, K., Okuzumi, S., \& Machida, M. N. 2015, ApJ, 801, 117

Ulrich, R. K. 1976, ApJ, 210, 377

Vorobyov, E. I. 2010, ApJ, 723, 1294

Whitney, B. A., Wood, K., Bjorkman, J. E., \& Wolff, M. J. 2003, ApJ, 591, 1049

Williams, J. P., \& Best, W. M. J. 2014, ApJ, 788, 59

Yen, H.-W., Koch, P. M., Takakuwa, S., et al. 2015, ApJ, 799, 193

Yorke, H. W., \& Bodenheimer, P. 1999, ApJ, 525, 330

Zhu, Z., Hartmann, L., Nelson, R. P., \& Gammie, C. F. 2012, ApJ, 746, 110 